\title{
Turbulence Modeling for Hypersonic Flows
}

\section{J. G. Marvin and T. J. Coakley}

(NASA-TM-101079) TURBULENCE MODELING FOR

N89-26181

HYPERSONIC FLOWS

Center) $49 p$

CSCL 200

Unclas

G3/34 0219613

June 1989

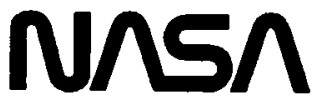

National Aeronautics and

Space Administration 
NASA Technical Memorandum 101079

\section{Turbulence Modeling for Hypersonic Flows}

J. G. Marvin

T. J. Coakley, Ames Research Center, Moffett Field, California

June 1989

\section{N/SN}

National Aeronautics and

Space Administration

Ames Research Center

Moffett Field, California 94035 


\section{SUMMARY}

Turbulence modeling for high-speed compressible flows is described and discussed. Starting with the compressible Navier-Stokes equations, methods of statistical averaging are described by means of which the Reynolds-averaged Navier-Stokes equations are developed. Unknown averages in these equations are approximated using various closure concepts. Zero-, one-, and two-equation eddy viscosity models, algebraic stress models, and Reynolds stress transport models are discussed. Computations of supersonic and hypersonic flows obtained using several of the models are discussed and compared with experimental results. Specific examples include attached boundary-layer flows, shock-wave boundary-layer interactions, and compressible shear layers. From these examples, conclusions regarding the status of modeling and recommendations for future studies are discussed.

\section{INTRODUCTION}

In this report we will discuss turbulence models that are used in numerical simulations of complex viscous flows. Although there are many applications and uses of turbulence models, we will restrict our attention primarily to high-speed compressible flows. The material covered constitutes a brief survey of the essential features of turbulence models and their status in applications, but does not include many details important in practice. For these, the reader is encouraged to consult the references.

Turbulence models are necessary in numerical simulations because of the impracticality of computing all scales of turbulent motion. Since these scales compose a range many orders in magnitude, the computer storage required to resolve all scales is much larger than the storage capacity currently available on the most powerful computers. Even if computers did exist with the required capacity, the computational speed of current computers is too slow to handle all but the simplest of problems. Thus approximate methods, or models of turbulence, are introduced to simplify and make the computations practical.

There are several approaches to turbulence modeling depending on how many of the turbulent scales are included in the modeling process. A more rigorous approach is to use subgrid-scale modeling (also known as large-eddy simulation) in which only turbulent eddies equal to or smaller than the numerical grid sizes are modeled. In this case the largest eddies are computed, and because they move and deform in time, the calculations are necessarily unsteady. This results in relatively large computing times and restricts the applicability of subgrid modeling to fundamental studies.

A more practical approach is to model all the scales of turbulent motion. The equations solved in this case are the Reynolds-averaged Navier-Stokes equations and the numerical solutions, which represent long time averages of the flow variables, are usually steady in time. This is the approach described here.

The report is organized into several main sections. It begins with a discussion of averaging procedures and the development of the Reynolds-averaged Navier-Stokes and related equations. After a brief discussion of the various types of turbulence models available, attention is directed to describing a representative sample of eddy viscosity models including explicit modifications to account for high speeds 
and compressibility. This is followed by a section on results in which representative computations are discussed and compared with experimental measurements. The paper concludes with a section on the current status of turbulence modeling for hypersonic flows with recommendations for future experiments and computation.

\section{REYNOLDS-AVERAGED NAVIER-STOKES EQUATIONS}

The basic differential equations used in numerical simulations are the Reynolds-averaged NavierStokes equations. These equations are derived from the compressible Navier-Stokes equations by an averaging process that will be described shortly. The time-dependent, compressible Navier-Stokes equations are written as follows:

$$
\begin{array}{rl}
\rho_{t}+\left(\rho u_{j}\right)_{j} & =0 \\
\left(\rho u_{i}\right)_{t}+\left(\rho u_{i} u_{j}+\sigma_{i j}\right)_{j}=0 & i=1,2,3 \\
(\rho E)_{t}+\left(\rho E u_{j}+u_{i} \sigma_{i j}+q_{j}\right)_{j} & =0
\end{array}
$$

where subscript notation has been used for partial derivatives, i.e., ()$_{t}=\partial / \partial t,()_{, i}=\partial / \partial x_{i}$, and the summation convention is used for repeated indices. The molecular stress tensor and heat flux vector are expressed as

$$
\begin{aligned}
\sigma_{i j} & =\delta_{i j} p-\mu\left(u_{i, j}+u_{j, i}-\frac{2}{3} \delta_{i j} u_{k, k}\right) \\
q_{j} & =-\kappa T_{j}=-\frac{\mu}{P r} h_{j}
\end{aligned}
$$

In these equations, $\rho$ is density, $u_{i}$ are Cartesian velocity components, $E$ is total specific energy, $T$ is temperature, $h$ is enthalpy, $e$ is internal energy, $P r$ is the Prandtl number, and the Stokes hypothesis is imposed. Assuming a perfect gas with constant specific heats, these variables are related as follows:

$$
\begin{aligned}
& p=(\gamma-1) p e \quad e=C_{v} T \quad h=C_{p} T \\
& \gamma=C_{p} / C_{v} \quad E=e+u_{i} u_{i} / 2 \quad \operatorname{Pr}=C_{p} \mu / \kappa
\end{aligned}
$$

In most applications, the Sutherland relation is used for molecular viscosity, i.e., $\mu=A T^{n} /(B+T)$, where $n, A$, and $B$ are constants that depend on the gas.

To derive the Reynolds-averaged Navier-Stokes equations an averaging operation is defined as follows:

$$
\bar{\rho}\left(x_{j}, t\right)=\frac{1}{2 T} \int_{t-T}^{t+T} \rho\left(x_{j}, s\right) d s
$$

where $2 T$ is the averaging interval, which is assumed to be large compared with the energy containing turbulent time scales, but small compared with the time scale of the mean or average motion. The mean density, $\bar{\rho}$, in this sense is a slowly varying function of time. Although $\bar{\rho}$ depends on the averaging interval, it is tacitly assumed that a range of values for $T$ exists for which $\bar{\rho}$ is practically independent of $T$ and it is this range that is applicable in the averaging operation. 
An alternate form of averaging which may be used in place of time-averaging is ensemble-averaging in which the averaging is performed over a large number of records or experiments. In this case the difficulty of selecting a time-averaging interval is not present and therefore this form of averaging is superior in many respects to time-averaging. Because results obtained using the two averaging forms are identical, we will continue to use the term time-averaging even though the preferred form is ensemble-averaging.

The fluctuating density, $\rho^{\prime}$, is defined as the difference between the density and its average value, i.e.,

$$
\rho^{\prime}=\rho-\bar{\rho}
$$

Averages and fluctuating quantities for other variables such as $p$ and $u_{i}$ are defined similarly. Although not strictly true unless $T \rightarrow \infty$, we assume here that $\overline{\rho^{\prime}}=0$ and $\overline{\bar{\rho}}=\bar{\rho}$, which is consistent with ensembleaveraging.

The time-averaged Navier-Stokes equations are obtained by averaging equation (1). The result involves averages such as $\overline{\rho u_{i} u_{j}}$ and $\overline{\rho h u_{j}}$, which can be split into averages of mean and fluctuation quantities, e.g.,

$$
\begin{aligned}
\overline{\rho u_{i} u_{j}} & =\overline{\left(\bar{\rho}+\rho^{\prime}\right)\left(\bar{u}_{i}+u_{i}^{\prime}\right)\left(\bar{u}_{j}+u_{j}^{\prime}\right)} \\
& =\bar{\rho} \bar{u}_{i} \bar{u}_{j}+\bar{\rho} \overline{u_{i}^{\prime} u_{j}^{\prime}}+\bar{u}_{i} \overline{\rho^{\prime} u_{j}^{\prime}}+\bar{u}_{j} \overline{\rho^{\prime} u_{i}^{\prime}}+\overline{\rho^{\prime} u_{i}^{\prime} u_{j}^{\prime}}
\end{aligned}
$$

For incompressible flows, where $\rho^{\prime}=0$, the last three terms in the above equation are absent. From this it is apparent that the compressible averaged equations will contain many more terms than the incompressible averaged equations. For this reason, an alternative form of averaging for velocity and energy variables has been developed, which leads to a form of the compressible averaged equations that is almost identical to the incompressible form. This is called mass-averaging (or Favre-averaging) in which the mean and fluctuating velocities and enthalpies are defined as follows:

$$
\begin{aligned}
\tilde{u}_{i} & =\overline{\rho u_{i}} / \bar{\rho}, & \tilde{h} & =\overline{\rho h} / \bar{\rho} \\
u_{i}^{\prime \prime} & =u_{i}-\tilde{u}_{i}, & h^{\prime \prime} & =h-\tilde{h}
\end{aligned}
$$

It is important to note that averages of fluctuating quantities are no longer zero, but finite, i.e.,

$$
\overline{u_{i}^{\prime \prime}}=-\overline{\rho^{\prime} u_{i}^{\prime}} / \bar{\rho}, \quad \overline{h^{\prime \prime}}=-\overline{\rho^{\prime} h^{\prime}} / \bar{\rho}
$$

but that mass-weighted averages of $u^{\prime \prime}$ and $h^{\prime \prime}$ are zero, i.e.,

$$
\overline{\rho u_{i}^{\prime \prime}}=\overline{\left(\bar{\rho}+\rho^{\prime}\right) u_{i}^{\prime \prime}}=0, \quad \overline{\rho h^{\prime \prime}}=\overline{\left(\bar{\rho}+\rho^{\prime}\right) h^{\prime \prime}}=0
$$

By introducing mean and fluctuating quantities into equation (1) and averaging, we obtain the massaveraged form of the compressible Navier-Stokes equations. These equations are written below. For simplicity, the bar and tilde notations have been omitted from averaged variables.

$$
\begin{array}{r}
\rho_{t}+\left(\rho u_{j}\right)_{j}=0 \\
\left(\rho u_{i}\right)_{t}+\left(\rho u_{i} u_{j}+\sigma_{i j}\right)_{j}=0 \quad i=1,2,3 \\
(\rho E)_{t}+\left(\rho E u_{j}+u_{j} \sigma_{i j}+q_{j}\right)_{j}=0 \\
\sigma_{i j}=\sigma_{i j}^{M}+\sigma_{i j}^{T}, \quad q_{j}=q_{j}^{M}+q_{j}^{T}
\end{array}
$$


where $\sigma_{i j}^{M}, \sigma_{i j}^{T}$, etc., are defined as

$$
\begin{array}{llrl} 
& \text { Reynolds stress tensor: } & \sigma_{i j}^{T} & =\overline{\rho u_{i}^{\prime \prime} u_{j}^{\prime \prime}}=\overline{\left(\bar{\rho}+\rho^{\prime}\right) u_{i}^{\prime \prime} u_{j}^{\prime \prime}} \\
& \text { Molecular stress tensor: } & \sigma_{i j}^{M} & =p \delta_{i j}-\mu\left(u_{i, j}+u_{j, i}-\frac{2}{3} \delta_{i j} u_{k, k}\right) \\
& \text { Reynolds heat-flux vector: } & q_{j}^{T} & =\overline{\rho h^{\prime \prime} u_{j}^{\prime \prime}}=\overline{\left(\bar{\rho}+\rho^{\prime}\right) h^{\prime \prime} u_{j}^{\prime \prime}} \\
& \text { Molecular heat-flux vector: } & q_{j}^{M} & =-\frac{\mu}{P r} h_{j} \\
& \text { Total energy: } & E & =e+k+u_{k} u_{k} / 2 \\
& \text { Turbulent kinetic energy: } & k & =\overline{\rho u_{k}^{\prime \prime} u_{k}^{\prime \prime}} / 2 \bar{\rho}
\end{array}
$$

In equation (11) it is assumed that $\mu$ is independent of time in the averages leading to $\sigma_{i j}^{M}$, and $q_{j}^{M}$.

The goal of turbulence modeling is to relate the Reynolds stresses and heat fluxes to known meanflow quantities such as velocity and temperature. This can be done in various ways which lead to different types of turbulence models. If the Reynolds stresses and heat fluxes are related algebraically to the meanflow variables, the corresponding models are called algebraic stress models. The most important and simplest subclass of these models are eddy viscosity models which relate Reynolds stresses to strain rates (or velocity derivatives) in a manner identical with molecular stresses. Eddy viscosity models will be the primary focus of this paper.

The simplest eddy viscosity models are the zero-equation models in which the eddy viscosity is modeled algebraically in terms of flow geometry and mean flow variables. More complicated turbulence models have been developed in which the Reynolds stresses are defined by field equations. These equations are derived by manipulating the Navier-Stokes equations for mean and fluctuating quantities (ref. 1). The resulting equation for the Reynolds stress tensor is given below

$$
\begin{array}{ll}
\text { Reynolds stress equation: } & \left(\sigma_{i k}^{T}\right)_{t}+\left(\sigma_{i k}^{T} u_{j}+q_{i k j}\right)_{j}=\rho\left(P_{i k}-\epsilon_{i k}\right) \\
\text { Production tensor: } & \rho P_{i k}=-\left(\sigma_{i j}^{T} u_{k, j}+\sigma_{k j}^{T} u_{i, j}\right) \\
\text { Dissipation tensor: } & \rho \epsilon_{i k}=-\left(\overline{\sigma_{i j}^{F} u_{k, j}^{\prime \prime}}+\overline{\sigma_{k j}^{F} u_{i, j}^{\prime \prime}}\right) \\
\text { Reynolds flux tensor: } & q_{i k j}=\left(\overline{\rho u_{i}^{\prime \prime} u_{k}^{\prime \prime} u_{j}^{\prime \prime}}+\overline{\sigma_{i j}^{F} u_{k}^{\prime \prime}}+\overline{\sigma_{k j}^{F} u_{i}^{\prime \prime}}\right) \\
\text { Fluctuating stress: } & \sigma_{i j}^{F}=\delta_{i j} p-\mu\left(u_{i, j}+u_{j, i}-\frac{2}{3} \delta_{i j} u_{k, k}\right)
\end{array}
$$

In these equations the fluctuating stress tensor, $\sigma_{i j}^{F}$, is interpreted to include both mean and fluctuating quantities. The dissipation tensor, $\rho \epsilon_{i k}$, contains both mean and fluctuating pressures and velocities and in this sense is a more general or extended definition than the conventional ones, which contain only fluctuating velocities. The production tensor is given directly in terms of the Reynolds stresses and mean velocity components, and thus requires no modeling. However, the dissipation and Reynolds flux tensors involve unknown averages and must be modeled. 
A simplified form of the Reynolds stress equation is obtained by taking its trace and is called the turbulent kinetic energy equation, or TKE equation. This equation is written below

$$
\begin{array}{ll}
\text { TKE equation: } & (\rho k)_{t}+\left(\rho k u_{j}+q_{k j}\right)_{j}=\rho(P-\epsilon) \\
\text { Production: } & \rho P=-\sigma_{i j}^{T} u_{i, j} \\
\text { Dissipation: } & \rho \epsilon=-\overline{\sigma_{i j}^{F} u_{i, j}^{\prime \prime}} \\
\text { TKE flux: } & q_{k j}=\frac{1}{2} \overline{\rho u_{k}^{\prime \prime} u_{k}^{\prime \prime} u_{j}^{\prime \prime}}+\overline{\sigma_{i j}^{F} u_{i}^{\prime \prime}}
\end{array}
$$

The TKE equation forms the basis of several classes of turbulence models, including the one- and two-equation models, and the algebraic stress models. In these models, the (square of the) velocity scale of turbulence is given by the TKE and a length scale of turbulence is given either algebraically or in terms of a field variable governed by an equation similar to the TKE equation.

\section{EDDY VISCOSITY MODELS}

Eddy viscosity models are the simplest turbulence models in the sense that they model turbulent stresses and fluxes by analogy to molecular stresses and fluxes. This approach is generally referred to as the Boussinesq approximation. The models may be expressed in terms of an eddy viscosity function, $\mu_{T}$, and a turbulent Prandtl number, $P r_{T}$, as follows:

$$
\begin{gathered}
\sigma_{i j}^{T}=\overline{\rho u_{i}^{\prime \prime} u_{j}^{\prime \prime}}=\frac{2}{3} \delta_{i j} \rho k-\mu_{T}\left(u_{i, j}+u_{j, i}-\frac{2}{3} \delta_{i j} u_{k, k}\right) \\
q_{j}^{T}=\overline{\rho h^{\prime \prime} u_{j}^{\prime \prime}}=-\frac{\mu_{T}}{P r_{T}} h_{j}, \quad P r_{T}=\frac{C_{p} \mu_{T}}{\kappa_{T}}
\end{gathered}
$$

where $\kappa_{T}$ is the turbulent conductivity. With these models, the whole problem of modeling is reduced to defining the eddy viscosity and turbulent Prandtl number. The turbulent Prandtl number is usually assumed to be a constant of the order of unity, but it may vary between classes of problems. (It is normally set equal to 0.9 for boundary-layer problems.) The eddy viscosity function may be expressed in terms of length and velocity scale functions, $l$ and $q$, as follows:

$$
\mu_{T}=\rho l q
$$

The way $l$ and $q$ are determined defines the type of eddy viscosity model to be used. If $l$ and $q$ are determined algebraically from mean flow data, the models are referred to as zero-equation models. If $l$ is determined algebraically, but $q$ is determined from a field equation such as the TKE equation, i.e., equation (13), the model is referred to as a one-equation model. If both $l$ and $q$ are determined from field equations, the resulting model is called a two-equation model. For this report we will discuss zero- and two-equation models. 
Eddy viscosity models were developed originally for incompressible flows and only later were extended to compressible flows. Aside from the use of mass-averaging instead of time-averaging, there is very little difference in form between the two types of models. This is because in the initial investigations, which were restricted to attached transonic and moderately supersonic flows, it was found that the incompressible forms were quite satisfactory. As we shall see, the extensions to higher-speed flows in some simple cases are satisfactory, but other more complex cases require specific corrections for compressibility effects.

\section{Zero-Equation Models}

Zero-equation models are the simplest of eddy viscosity models in the sense that they do not make use of additional field equations. In this section we will discuss two widely used models that are representative of most other zero-equation models and that will be discussed in the results section. Unless otherwise stated, it is assumed that these models are applied at solid walls using no-slip boundary conditions

Cebeci-Smith Model. - The model described here is a simplified version of the model described in reference 2. It is a two-layer model that uses Prandtl's mixing length model (ref. 3) for the inner layer and Clauser's model (ref. 4) for the outer layer. The model is expressed as follows:

$$
\begin{gathered}
\mu_{T}=\min \left(\mu_{T I}, \mu_{T O}\right) \\
\mu_{T I}=\rho l^{2} s, \quad \mu_{T O}=0.0168 \rho \delta_{i}^{*} u_{e} / I \\
l=0.4 y d, \quad d=1-\exp \left(-y^{+} / A^{+}\right) \\
u_{\tau}=\sqrt{\tau_{w} / \rho_{w}}, \quad y^{+}=u_{\tau} y / \nu_{w} \\
\delta_{i}^{*}=\int_{0}^{\delta}\left(1-u / u_{e}\right) d y, \quad I=1+5.5(y / \delta)^{6}
\end{gathered}
$$

the strain-rate parameter, $s$, is usually taken to be the shearing strain, $\left|u_{y}+v_{x}\right|$, for two-dimensional problems. The operation of taking the minimum in equation (16) is interpreted to mean using the inner eddy viscosity, $\mu_{T I}$, until it first becomes larger than the outer eddy viscosity, $\mu_{T O}$, beyond which point the outer formula is used exclusively. Parameter I is Klebanoff's intermittency factor, $u_{\tau}$, is the friction velocity, $\tau_{w}$, is the wall shear, $\nu_{w}=\mu_{w} / \rho_{w}$, and the subscript $w$ indicates wall values. The nondimensional parameter $A^{+}$, from van Driest (ref. 5) generally depends on the streamwise pressure gradient and surface blowing and roughness characteristics (ref. 2). For boundary layers with smooth solid walls and zero or small pressure gradients, $A^{+}$is a constant, i.e.,

$$
A^{+}=26
$$

A more complicated and general version of this model including transition modeling terms is given in reference 2.

Baldwin-Lomax Model. - The Baldwin-Lomax (B-L) turbulence model (ref. 6) is similar to the Cebeci-Smith (C-S) model, but it incorporates features that make it more advantageous for complex 
two- and three-dimensional flows. It is similar to the (C-S) model in that it uses nearly the same inner model, but it differs with respect to the outer model. This model can be expressed as follows:

$$
\begin{gathered}
\mu_{T}=\min \left(\mu_{T I}, \mu_{T O}\right) \\
\mu_{T I}=\rho l^{2} s, \quad s=\sqrt{\omega_{i} \omega_{i}} \\
\mu_{T O}=0.027 \rho y_{\max } \min \left(F_{\max }, 0.25 u_{D}^{2} / F_{\max }\right) / I \\
F=y s d, \quad u_{D}=|\vec{u}|_{\max }-|\vec{u}|_{\min }, \quad \delta=y_{\max } / 0.3
\end{gathered}
$$

where $y_{\max }$ is the outermost value of $y$ in the boundary layer where $F$ has a local maximum, $F_{\max }$. In these formulas $l$ is the Prandtl mixing length given by equation (16), $d$ is the Van Driest damping factor, and $I$ is the intermittency factor of equation (16) in which $\delta$ is replaced by $y_{\max } / 0.3$ as indicated.

It should be noted that with the B-L model, in contrast with the C-S model, the strain-rate function, $s$, is defined as the magnitude of the vorticity vector and not the shearing strain. This makes the model directly applicable to three-dimensional problems where an invariant shearing strain is not well defined.

A basic advantage of this model over the C-S model is a result of how the outer model is defined. Referring to equation (16) we see that the C-S model requires both the displacement thickness, $\delta_{i}^{*}$, and the boundary-layer thickness, $\delta$, which is used in the intermittency function. Both of these thickness parameters frequently are not well defined and are difficult to compute, especially for separated flows. The advantage of the B-L model is that it uses a length scale, $y_{\max }$, which is well defined and easily computed for a wide class of flows. This does not necessarily mean that the B-L model is superior to the C-S model on a physical basis, but it does mean that it is more convenient on a numerical basis.

From a physical standpoint, it has been found that the C-S and B-L models give similar predictions of both attached and separated boundary-layer flows for low to moderate supersonic flows. Predictions of attached flows are usually in good agreement with experiments, but predictions of separated flows are frequently deficient. At hypersonic speeds, the models also tend to give similar predictions, although there is some evidence that the B-L model may be more sensitive to Mach number than the C-S model is.

The procedure of applying no-slip boundary conditions is frequently referred to as the integration-tothe-wall procedure. To be applicable, the numerical mesh spacing normal to the wall must be chosen such that the value of $y^{+}$at the first point off the wall is of the order of unity, placing it well within the viscous sublayer.

For some numerical algorithms, such as explicit methods, the procedure of integrating to the wall has detrimental effects on numerical stability because of the fine mesh spacing required. In this case an alternate approach called the law-of-the-wall procedure, or wall-function method, is used. In this approach a slip-type boundary condition, based on the logarithmic velocity law of turbulent boundary layers, is used. This law can be written (for incompressible flow) as

$$
\begin{gathered}
u=\frac{u_{\tau}}{\kappa} \ln E y^{+} \\
\kappa=0.4, \quad E=9.128
\end{gathered}
$$


To apply this method, within the context of time-marching Navier-Stokes solvers, the above formula for velocity is solved (by a Newton-Raphson procedure) for the friction velocity, $u_{\tau}$, using for $u$ and $y$ their values at the first point off the wall. The values of $\nu_{w}$ and $\rho_{w}$ are obtained by extrapolating the temperature to the wall. Once $u_{\tau}$ is determined, the wall shear stress, $\tau_{w}$, is obtained, which is then used directly in the boundary condition for wall velocity. More complicated formulas have been developed for compressible flows and flows involving wall heat transfer. ${ }^{7}$

\section{Two-Equation Models}

Zero-equation models are well adapted to simple attached flows where a single well defined shear layer is easily identified. There are many complex flows where this is not the case, however, and use of zero-equation models becomes difficult or unwieldy. Examples of such flows include separated flows behind bluff bodies and multiple intersecting shear layers. In these cases it is difficult to define appropriate velocity and length scales because several such scales are usually present in the flow. For this reason, more advanced models have been developed in which the velocity and length scales are determined from field equations. These are the two-equation eddy viscosity models.

The prototype field equation for the two-equation models is the turbulent kinetic energy equation, equation (13). In order to use this equation, averages or correlations in the dissipation and TKE flux terms must be modeled in terms of known or mean-flow quantities. It is beyond the scope of this paper to explain in detail how these terms are modeled. Instead, we will simply discuss the results of the modeling.

There are essentially two terms in the TKE equation that must be modeled. These are the TKE flux, $q_{k i}$, and the dissipation, $\epsilon$. For the TKE flux, a gradient-diffusion approximation is used, i.e.,

$$
q_{k j}=-\left(\mu+\frac{\mu_{T}}{P r_{k}}\right) k, j
$$

where $\mathrm{Pr}_{k}$ is a modeling constant (Prandtl number) of the order of unity.

The absolute dissipation rate, $\epsilon$, is obtained from a separate field equation similar to the TKE equation given below. The velocity and length scales, and the eddy viscosity, are expressed in terms of $k$ and $\epsilon$ as follows

$$
\begin{gathered}
\mu_{T}=C_{\mu} f \rho q l=C_{\mu} f \rho k / \omega=C_{\mu} f \rho k^{2} / \epsilon \\
q=\sqrt{k}, \quad l=q / \omega, \quad \omega=\epsilon / k
\end{gathered}
$$

where $f$ is a damping function analogous to the van Driest damper, equation (16), $C_{\mu}$ is a modeling constant, and $\omega$ is the specific dissipation rate. With these approximations, the TKE equation can be written

$$
\begin{gathered}
(\rho k)_{t}+\left(\rho k u_{j}+q_{k j}\right)_{j}=\rho(P-\epsilon) \\
\rho P=-\sigma_{i j}^{T} u_{i, j}=\mu_{T} S-\frac{2}{3} \rho k D \\
S=\left(u_{i, j}+u_{j, i}\right) u_{i, j}-\frac{2}{3} u_{k, k}^{2}, \quad D=u_{k, k}
\end{gathered}
$$


In these equations, $P$ is the turbulent production that is reexpressed in terms of the eddy viscosity and the strain invariants $S$ and $D$. For incompressible flows the dilatation, $D=u_{k, k}$, is zero, but for compressible flows this term is nonzero and can be an important modeling term in some cases.

The term on the right-hand side of equation (21) is a turbulence source function that may be expressed in terms of a nondimensional source function, $h_{k}$, using equations (20) and (22), i.e.,

$$
\begin{gathered}
\rho(P-\epsilon)=h_{k} \rho \omega k \\
h_{k}=C_{\mu} f \frac{S}{\omega^{2}}-\frac{2}{3} \frac{D}{\omega}-1
\end{gathered}
$$

Equation (21) is the prototype field equation for all two-equation models. With general two-equation models, the variables $k$ and $\epsilon$ (or $\omega$ ) of equation (20) are expressed in terms of two auxiliary variables, $s_{1}$, and $s_{2}$, each of which is governed by field equations similar to equation (21). This general form of a two-equation model can be written as follows:

$$
\begin{gathered}
k=k\left(s_{1}, s_{2}\right), \quad \epsilon=\epsilon\left(s_{1}, s_{2}\right) \\
\left(\rho s_{i}\right)_{t}+\left(\rho s_{i} u_{j}+q_{i j}\right)_{j}=h_{i} \rho \omega s_{i} \quad i=1,2 \\
h_{i}=C_{i 1}\left(C_{\mu} f \frac{S}{\omega^{2}}-\frac{2}{3} \frac{D}{\omega}\right)-C_{i 2} \\
q_{i j}=-\left(\mu+\frac{\mu_{T}}{P r_{i}}\right) s_{i, j}
\end{gathered}
$$

In these equations there is no summation on the index $i$.

The eddy viscosity damping function, $f$, is usually expressed in terms of a turbulence Reynolds number, $R_{T}$, which in turn is written in terms of $k$ and $\epsilon$ (or $\left.\omega\right)$. Typical expressions for $f$ and $R_{T}$ are

$$
f=1-\exp \left(-\alpha R_{T}\right), \quad R_{T}=\frac{\rho k}{\mu \omega}
$$

where $\alpha$ is a constant.

For fully developed turbulent flows, the turbulent Reynolds number becomes very large and the damping function approaches unity. On the other hand, at low Reynolds numbers (e.g., in laminar regions or the viscous sublayer) $f$ goes to zero. In general, the variables $C_{i 1}$ and $C_{i 2}$ of equation (24) are also functions of the turbulence Reynolds number, analogous to equation (25), although in some cases they may involve additional terms. The Prandtl numbers $P r_{1}, P r_{2}$ are usually taken to be constant. At large values of the turbulence Reynolds number the variables $C_{i 1}$ and $C_{i 2}$ generally approach constant values along with the damper $f$. 


\section{1. $k-\epsilon$ Model}

One of the most widely used two-equation models is the $k-\epsilon$ model originated by Launder and Spalding (ref. 8). In this model $s_{1}=k$ and $s_{2}=\epsilon$. The high Reynolds number form of the constants $C_{i 1}$ and $C_{i 2}$, as well as the other constants in equation (24), are given by

$$
\begin{aligned}
C_{\mu}=0.09, & C_{11}=C_{12}=f=P r_{1}=1 \\
C_{21}=1.45, & C_{22}=1.92, \quad P r_{2}=1.3
\end{aligned}
$$

These constants have been obtained by comparing solutions of the governing equations with experimental results. For example, the constants $C_{11}$ and $C_{12}$ come directly from the TKE equation. The constant $C_{22}$ is determined from experiments on the decay of isotropic turbulence in which case all production and diffusion terms are absent from the equations, and an exact solution is easily obtained. The other constants are determined by obtaining approximate solutions for the wall region of equilibrium boundary layers, where $P=\epsilon$, and the logarithmic law, equation (18), is applicable, and by numerically optimizing free shear flow solutions.

The high Reynolds number form of the $k-\epsilon$ model described above is applicable to fully developed turbulent flows and does not apply to the viscous sublayer. For such applications, the molecular viscosity is much smaller than the turbulent viscosity and usually is neglected in the diffusion fluxes. In these cases, however, special slip-type boundary conditions based on equation (18) must be applied to the velocity and turbulence variables because the first numerical grid point must be taken well outside the viscous sublayer (in the fully turbulent region) and no-slip conditions are inappropriate. This approach has been followed by Launder and Spalding (ref. 8) and others. The generalization to compressible flow is described by Viegas, Rubesin and Horstman (ref. 7). Although this approach is convenient for many problems it is not easily adapted to low Reynolds number flows where transition phenomena are important. In these cases, a more general low Reynolds number form of the model must be used in which $C_{11}, C_{12}$, etc., depend on $R_{T}$. Several such models have been developed, including those by Jones and Launder (ref. 9), Chien (ref. 10), and Wilcox and Rubesin (ref. 11). Because the formulas defining these models are relatively complicated, we will not give them here. Instead, we will describe an alternative low Reynolds number, two-equation model that is given below.

2a. $q-w$ Model a

The $q-\omega$ model was developed to overcome numerical stability problems encountered with several low Reynolds number, two-equation models (refs. 9, 10, and 11). A discussion of these problems, and the development of the $q-\omega$ model, is given in references 12,13 , and 14. For this model, the variables $s_{1}$ and $s_{2}$ of equation (24) are taken as

$$
s_{1}=q=\sqrt{k}, \quad s_{2}=\omega=\epsilon / k
$$

The parameters and constants in the equations are given by the following relations: 


$$
\begin{array}{cc}
C_{\mu}=0.09, \quad f=1-\exp (-0.02 q y / \nu) \quad, \quad P r_{1}=P r_{2}=2 \\
C_{11}=C_{12}=0.5, \quad C_{21}=0.055+0.5 f \quad, \quad C_{22}=0.833, \quad C_{23}=\frac{2}{3} \\
h_{2}=C_{21}\left(C_{\mu} \frac{S}{\omega^{2}}-C_{23} \frac{D}{\omega}\right)-C_{22}
\end{array}
$$

Numerical boundary conditions to be applied with this model at solid walls are given by $u=v=q=\omega_{y}=0$.

\section{2b. $q-\omega$ Model b: Compressibility correction}

The previous model was tested on an oblique shock-wave boundary-layer interaction flow for a separated case, but it failed to predict any separation (ref. 15). In reference 15, a correction to the model was introduced that led to substantially improved predictions. This modification was based on the work of Morel and Monsour (ref. 16) who observed that in a uniaxial compression, the standard $k-\epsilon$ model predicts that the turbulence length scale should increase, which runs counter to the physical expectation that it should decrease. Arguing that the product of the density and the turbulent length scale should remain constant in a uniaxial compression, they derived a correction to the source term of the $\epsilon$ equation. Translated to the $\omega$ equation, this modification results in a new value of the constant multiplying the dilatation term, i.e.,

$$
C_{23}=2.4
$$

In reference 17 , Vandromme proposed a compressibility modification with some similarities to the modification described here. His modification was based on earlier work by Rubesin and included density gradient terms as well as dilatation terms. Results using this model will be reported in the section on compressible shear layers.

\section{2c. $q-w$ Model c: Heat transfer correction}

The previously described correction for compressibility improves the predictions of pressure distribution and separation, but the surface heat transfer remains relatively unaffected and too high in the region of reattachment. To remedy this difficulty, a modification or constraint on the turbulent length scale was imposed, following the work of Monsour reported in Kline, Cantwell, and Lilley (ref. 18). In this correction, an upper bound is placed on the length scale appearing in the eddy viscosity such that it can never be greater than a constant times the Prandtl length scale in the wall region. The result is

$$
\ell=\min (2.4 y, q / \omega)
$$

This correction generally does not change the predictions of the turbulence model exept near a reattachment point, and, to a lesser extent, near a separation point. This occurs because in equilibrium or attached flows, the turbulent length scale $q / \omega$ is approximately equal to $2.4 \mathrm{y}$ in the wall region. This model will be referred to as the $q-\omega$ model $\mathrm{c}$. 


\section{RESULTS}

The status of modeling for high-speed flows will now be described by comparing predictions with the results of experiments. Experimentation plays an important role in the development of turbulence models by providing data on the flow physics required to substantiate modeling assumptions and in verifying the performance of models in testing. Wherever possible, experimental data from low- and high-speed flows will be contrasted to illustrate similarities and differences. Emphasis will be on attached flows, shock-wave boundary-layer interaction flows, and shear layers. References 19, 20, and 21 cite data that have been used to evaluate turbulence models for aerodynamic flow predictions.

\section{Attached Boundary-Layer Flows}

Modeling for hypersonic attached flows is more mature than for the other flows we consider. Eddy viscosity models perform reasonably well, as our examples will show. This fact may not be surprising because the modeling has been founded on a rather substantial experimental data base used together with knowledge regarding the behavior of incompressible flows.

Figure 1 shows a composite sketch of a turbulent boundary layer constructed from a substantial incompressible data base. Velocity profile data can be collapsed onto a single curve using the friction velocity, $u_{\tau}$, as a scaling parameter. Regions of the viscous sublayer, the logarithmic region, and the outer layer are depicted. The viscous sublayer is the region where molecular viscosity is important. It consists of a laminar sublayer region and a buffer region that blends with the logarithmic turbulent region. The logarithmic region is characteristic of all turbulent boundary layers and can be expressed as a function of the Reynolds number based on the friction velocity, or $y^{+}$. The outer region, which actually begins quite close to the wall $(y / \delta$ between 0.1 and 0.2 ), is characterized by a wake-like region whose shape and thickness depend on the pressure gradient imposed by the outer inviscid flow field and the Reynolds number.

At the high speeds associated with supersonic and hypersonic Mach numbers, similar experimental observations have been made. In these cases, however, it is necessary to introduce a compressiblity transformation (ref. 22) to adjust the profiles appropriately. Figure 2 shows the transformed velocity profiles taken in a very high Mach number helium wind tunnel. It should be noted that at very high Mach numbers, the pressure gradient must balance turbulent normal stresses arising form the normal momentum balance. Also, the sublayer becomes thicker as the Mach number increases.

Representing turbulent velocity profiles using log-law variables enabled integration of the mean momentum equation to determine such quantities as skin friction and heat transfer. But, with the advent of finite difference methods for solving the boundary-layer equations, the development of mixing length and eddy viscosity models was facilitated when it was experimentally observed that the shear stress distribution across a boundary layer changed little because of compressibility. Figure 3, taken from Sandborn (ref. 23), shows compressible data up to Mach 7, compared with similar data representative of incompressible flows. Earlier, Maise and McDonald (ref. 24), using a similar approach with adiabatic wall temperature data, 
showed that the mixing length and eddy viscosity, scaled by the boundary-layer thickness and the incompressible displacement thickness, respectively, were essentially independent of compressibility effects up to a Mach number of 5 . See figures $4 a$ and $4 b$.

The effects of compressibility and wall temperature on skin friction are shown in figures $5 \mathrm{a}$ and $5 \mathrm{~b}$. The solid line is the van Driest correlation based on the Karman-Schoener incompressible friction law and represents the available skin friction data to within $10 \%$ for the adiabatic wall data and to within $20 \%$ for the data with heat transfer. See Hopkins and Inouye (ref. 25). Computations using the boundary-layer equations are compared with the data in the figure. Aside from showing that eddy viscosity models predict the correct influence of compressibility on skin friction (fig. 5a), several other conclusions can be reached. The choice of mass-averaging or time-averaging has no significant effect on the predicted results. The zeroequation C-S model reproduces the van Driest result somewhat more accurately than do the other models; thus this model would have to be the choice for prediction, considering its simplicities. The effects of heat transfer are illustrated in figure $5 \mathrm{~b}$ where a two-equation model prediction is compared with the van Driest correlation for $M=5$. The result, which is typical of most eddy viscosity predictions, deviates from the van Driest variation as total temperature ratio decreases and points to a caution regarding accurate prediction of cool-wall heat transfer trends, although the data are considerably scattered in these cases.

Shang (ref. 26) extended computations using the C-S model to higher Mach numbers. He incorporated the normal momentum equation to account for nonzero normal pressure gradients and, more importantly, accounted for triple correlations involving density fluctuations usually omitted at lower Mach numbers. Results are shown in figures 6 and 7. Data and computations from two models, one with density fluctuation terms and one without density fluctuation terms, are compared. The inclusion of these terms affects the heat-transfer predictions somewhat more than the skin friction, but either approach produces reasonably accurate results, considering the uncertainties in the data.

It is interesting to note that at lower Reynolds numbers the data tend to be underpredicted, particularly the heat transfer. Such results are common because boundary layer transition influences the region encompassed by the low Reynolds number. These influences also tend to affect data correlation and may explain why there is more scatter in the cold-wall data around the van Driest predictions at higher Mach numbers where transition lengths are substantial. Figure 7, from Shang (ref. 26), shows skin-friction measurements for low and high Reynolds numbers compared with computations obtained with and without accounting for density fluctuation effects. At high Reynolds numbers, where the turbulent flow is fully developed, the computations compare reasonably well with the data, although it is difficult to conclude whether it is necessary to include the density fluctuation effects because of the data scatter. At low Reynolds numbers, the data are scattered for all Mach numbers, but this is especially pronounced at the highest Mach numbers, probably because of transition effects. The computations show poorest agreement at the high Mach numbers, so a cautionary note is made for this regime.

The effects of low Reynolds numbers can be accounted for at low Mach numbers approximately by modifying either the maximum mixing length or the outer eddy viscosity used in the model formulations. (See McDonald, ref. 27). Bushnell (refs. 28, 29, and 30) investigated the low Reynolds number problem for high Mach numbers and provided a data analysis which indicated that such low-speed, low Reynolds number corrections could still be applied at high Mach numbers. However, it was necessary to define a different Reynolds number. He recommended $\delta^{+}$, the Reynolds number based on the friction velocity, wall density, and boundary-layer thickness. Figure 8 , taken from reference 30 , shows the domain of importance 
for including low Reynolds number effects. For values of $\delta^{+}$below 3000, the effects become more important and, in particular, below 400, they are significant. Lines of constant Mach number indicate that low Reynolds number effects can become substantial at high Mach numbers even though the length Reynolds number is large.

Modeling for adverse pressure gradient flows at hypersonic Mach numbers is less well advanced because the data base is limited. Figure 9 presents a list of experiments and pertinent test variables. It represents a partial, but representative, list of benchmark flows available for model evaluation. Mach number is limited to 7 and the wall-to-total-temperature range is mostly adiabatic. For these representative flows, eddy viscosity models give reasonably accurate results. Typical comparisons between computations and experiments for the skin friction, taken from reference 19, are shown in figure 10. The C-S model with the pressure gradient correction (i.e., the $p^{+}$term) and the higher-order eddy viscosity and Reynolds stress models all adequately predict the influence of pressure gradient over a wide range of Reynolds numbers.

\section{Shock-Wave Boundary-Layer Interaction Flows}

In this section we discuss several examples of shock-wave boundary-layer interaction flows, some of which are separated. Figure 11, taken from reference 21, summarizes the status of experiments and computation for a variety of compressible flows. We will discuss a limited number of these flows consisting of both supersonic and hypersonic cases.

Figure 12 illustrates the experimental geometry of two hypersonic flows to be discussed. The first is a Mach 7 flow about an ogive-cylinder geometry. Two subcases of this flow will be considered: the first is the flow over the clean body from the nose rearward, and the second consists of the shock-wave boundarylayer interaction on the cylinder produced by a $15^{\circ}$ ring-shock generator. Figure 13 shows comparisons of predictions and measurements of surface properties for the clean-body case (ref. 15). Three predictions are shown, one corresponding to laminar flow and the other two corresponding to turbulent flow obtained using the C-S and $q-\omega, a$ models. Transition was enforced in the modeling at a location about $10 \mathrm{~cm}$ from the nose. It is apparent that both turbulence models accurately predict surface pressure, skin friction, and heat transfer (Stanton number) distributions.

Results of predictions and measurements of the shock-wave boundary-layer interaction flow on the cylinder are shown in figure 14. In this case, measurements of surface-pressure, skin-friction, and heattransfer are compared with predictions made with the C-S, B-L, and the three versions of the $q-\omega$ model. It is apparent from these results that both the zero-equation models and the unmodified $q-\omega, a$ models strongly overpredict the peak pressure in the interaction (fig. 14a). This basically is the result of the inability of the three models to adequately predict the extent of separation, which is indicated by the plateau in the measured pressure distribution ahead of the interaction. Substantial improvement was obtained with the $q-\omega$ model $b$, which incorporated the compressibility correction. Results obtained with the $q-\omega, c$ model, which incorporated the modification for heat transfer, were similar to those for model $b$.

Skin-friction and heat-transfer distributions for this case are shown in figures $14 \mathrm{~b}$ and $14 \mathrm{c}$. It is apparent from these results that although the two zero-equation models give reasonable predictions of peak 
heating and skin-friction, their predictions in the region of separation are less accurate. Predictions made with the unmodified $q-\omega, a$ model indicate no separation at all and, as a result, grossly overpredict both peak heating and skin-friction. The computation made with the $q-\omega, b$ model shows an improvement in skin-friction prediction, but still strongly overpredicts peak heating. Finally, the prediction made with the $q-\omega, c$ model produced results that were in reasonably good agreement with the measurements.

Computed and measured pressure contours of the flow are shown in figures 15 and 16 . Figure 15 shows measured pressure contours compared with contours obtained with the $q-\omega, c$ model. It is apparent that the overall features of the flow are well predicted by the model. Figure 16 compares predictions obtained with the $q-\omega, c$ and the B-L models. This result illustrates that the differences in model predictions of surface characteristics are accurately reflected in predictions of flow-field variables as well.

Calculations and measurements of the $7.5^{\circ}$ shock generator case are also discussed in reference 15. In this case the flow is attached and the predictions of the two zero-equation models and the $q-\omega, c$ model give similar results that are in good agreement with the experiment.

The second flow is a compression corner flow, also illustrated in figure 12. Results of measurements and computation are shown in figures 17 and 18, corresponding to attached and separated cases, respectively (ref. 15). Calculations of surface pressure and heat transfer for the attached flow case (fig. 17), made with the C-S, B-L, and $q-\omega, c$ models, indicate reasonably good agreement between computation and experimentation. In the separated case (fig. 18) the predictions are also in reasonable agreement, although the pressure plateau and extent of separation predicted by the $q-\omega$ model is better than that predicted by the B-L model. In the reattachment zone, both models underpredict overshoots in measured pressure and heat-transfer distributions. In this case, computations with the C-S model were unreliable because of difficulties in computing boundary-layer and displacement thickness distributions, and are therefore not shown.

It should be noted that the modifications made to the $q-\omega$ model were general in the sense that no arbitrary constants were introduced and then adjusted to improve predictions. Furthermore, the modifications introduced did not interfere with or change predictions of simple attached flows (e.g., the clean-body flow). This is the type of modification one seeks when improving turbulence models for complex flows.

The final shock-wave boundary-layer interaction to be discussed is a Mach-3 compression corner flow illustrated in figure 19. Calculations of this flow with a corner angle of $20^{\circ}$ are compared with results of the experiment in figure 20 , and are discussed in greater detail in reference 7 . The turbulence model used in this case was the Jones-Launder $k-\epsilon$ model. Two wall treatments were investigated, namely, the integration-tothe-wall and wall-function procedures. In this $\left(20^{\circ}\right)$ case, the flow was mildly separated. When predictions and measurements are compared, it is clear that noticeable differences in predictions result from different wall treatments using the same model. From both skin-friction and pressure distributions it is clear that the wall function treatment gives better predictions of separation and surface pressure. In addition, it also gives much better agreement with downstream skin-friction distributions than does the integration-to-the-wall procedure. Results similar to these were also observed in the $16^{\circ}$ and $24^{\circ}$ cases (ref. 7).

The primary reason for the differences between model predictions in this case lies in the low Reynolds number (damping) terms of the Jones-Launder model that strongly influence results when the integrationto-the-wall procedure is used, but that are inactive when the wall function procedure is used. Although the 
low Reynolds number terms can produce accurate results for zero pressure gradient attached flows, it is apparent that unless they are chosen carefully they can lead to unreliable predictions of complex flows.

\section{Compressible Shear Layers}

Knowledge of the physics of high-speed shear layers is limited at present. Experiments have shown that the far-field spreading angle, a measure of mixing, is reduced considerably, compared to that for incompressible flows. Figure 21 illustrates the status. The inverse of the spreading angles obtained from various experiments on single-stream mixing layers are shown as a function of Mach number. Reduction in spreading angle by a factor of 3 occurs at Mach 5 . Various postulates to explain this reduced mixing have been proposed, but experimental evidence to substantiate them is lacking.

Application of incompressible turbulence models, extended to account for compressibility as described in previous sections, fails to produce accurate predictions of the spreading rate. The line labeled $k-\epsilon$ represents such a prediction. In reference 31 , Dash et al. proposed a new model to account for compressibility in free shear layers and predicted the spreading angle as shown by the symbols labeled $k-\epsilon, c c$. The compressibility correction (cc) involved the empirical function $K\left(M_{\tau}\right)$, shown in figure 22, where $M_{r}$ is the ratio of the square root of the turbulent kinetic energy to the speed of sound, $k^{1 / 2} / a$, at the point of maximum $k$ in the layer.

It is noteworthy that the modification also gives reasonable predictions for two-stream supersonic mixing. Figure 23 shows a comparison of $k-\epsilon$ model predictions compared with the measurements of Chinzie (ref. 32). The inverse of the two-stream spreading rate, scaled by the spreading rate $\sigma_{0}$ for which one stream is stationary, compares reasonably well with the $k-\epsilon, c c$ model prediction. Deviation of the data from the modified model prediction for the higher second-stream Mach numbers may be a result of free-stream turbulence present in the experiment.

Vandromme (ref. 17) also reported successful predictions of the single-stream spreading rate. As mentioned in the section on modeling, he used the ideas of Rubesin to make compressibility corrections to the turbulent kinetic energy and dissipation equations of the $k-\epsilon$ model. The results of his predictions are shown compared with results of experiments in figure 24 . Substantial agreement was achieved.

More work will be necessary before the compressible mixing layer problem can be considered solved. Current modeling modifications are, to a considerable extent, ad hoc and have not been verified for a wide range of cases. Futhermore, they are not based on an understanding of the physical mechanisms involved. To understand these mechanisms, more experimentation is needed. It should also be noted that research is underway at Ames Research Center to use full simulations of compressible shear layers using the timedependent Navier-Stokes equations to provide more complete information on mixing phenomena. It is hoped that this research will lead to improved modeling of compressible shear flows. 


\section{CONCLUDING REMARKS}

In the preceding paragraphs we have described the development and status of turbulence models used in the numerical simulation of complex hypersonic flows. In our discussion we emphasized eddy viscosity models which constitute the simplest but most widely used class of turbulence models. Two subgroups of models were discussed-zero-equation and two-equation models. Each of these models has theoretical advantages over the other. For example, two-equation models provide a more general specification of turbulent length and velocity scales than zero-equation models, but they often display numerical stability problems which are not common to zero-equation models.

The basic models discussed are similar to those developed originally for incompressible flow. This is because in many applications, especially to simple attached boundary-layer flows at low to moderate supersonic speeds, the incompressible forms give satisfactory results. As discussed in the text, however, there is evidence that these incompressible forms become unsatisfactory as the flow complexity and/or the Mach number increase. With respect to flow complexity, it was shown that compressibility corrections were necessary to give satisfactory predictions of several hypersonic shock-wave boundary-layer interaction flows. With respect to Mach number, it was shown that incompressible model forms are unsatisfactory. for compressible free-shear flows. In this case, too, compressibility corrections could be found which lead to satisfactory predictions.

The status of turbulence modeling for hypersonic flows is still far from complete, however. More experimental data and computational comparisons will be necessary to verify and establish the compressibility corrections made to date. In addition, more experimental and computational work will be needed, especially at low Reynolds numbers, because this flow regime is more prevalent at hypersonic speeds, and because the available data base in this case is still quite limited. 


\section{REFERENCES}

1. Rubesin, M. W.; and Rose, W. C.: The Turbulent Mean Flow, Reynolds-Stress, and Heat-Flux Equations in Mass-Averaged Dependent Variables, NASA TM X-62248, 1973.

2. Cebeci, T. and Smith, A. M. O.: Analysis of Turbulent Boundary Layers. Academic Press, 1974.

3. Prandtl, L.: The Mechanics of Viscous Fluids, in Aerodynamic Theory, Vol. III, Durand, W. F. (Ed.), Springer-Verlag, Pasadena, CA, 1943.

4. Clauser, F. H.: The Turbulent Boundary Layer. Advances in Applied Mechanics, Vol. IV, Academic Press, 1956.

5. van Driest, E. R.: On Turbulent Flow near a Wall. J. Aeronaut. Sci., vol. 23, 1956, pp. 1007-1011.

6. Baldwin, B. S.; and Lomax, H.: Thin Layer Approximation and Algebraic Model for Separated Turbulent Flows. AIAA Paper 78-257, Jan. 1978.

7. Viegas, J. R.; Rubesin, M. W.; and Horstman, C. C.: On the Use of Wall Functions as Boundary Conditions for Two-Dimensional Separated Compressible Flows. AIAA Paper 85-0180, Jan. 1985.

8. Launder, B. E.; and Spalding, D. B.: Mathematical Models of Turbulence. Academic Press, 1972.

9. Jones, W. P.; and Launder, B. E.: The Prediction of Laminarization with a Two-Equation Model of Turbulence. Intern. J. Heat Mass Transfer, vol. 15, 1972, pp. 301-304.

10. Chien, K. Y.: Predictions of Channel Boundary-Layer Flows with a Low-Reynolds-Number Turbulence Model. AIAA J., vol. 20, Jan. 1982, pp. 33-38.

11. Wilcox, D. C.; and Rubesin, M. W.: Progress in Turbulence Modeling for Complex Flow Fields Including the Effects of Compressibility. NASA TP-1517, 1980.

12. Coakley, T. J.: Turbulence Modeling Methods for the Compressible Navier-Stokes Equations. AIAA Paper 83-1693, July 1983.

13. Coakley, T. J.: A Compressible Navier-Stokes Code for Turbulent Flow Modeling. NASA TM-85899, 1984.

14. Coakley, T. J.; and Hsieh, T.: Comparison between Implicit and Hybrid Methods for the Calculation of Steady and Unsteady Inlet Flows, AIAA Paper 85-1125, July 1985.

15. Voung, S. T.; and Coakley, T. J.: Modeling of Turbulence for Hypersonic Flows with and without Separation. AIAA Paper 87-0286, Jan. 1987.

16. Morel, T.; and Mansour, N. N.: Modeling of Turbulence in Internal Combustion Engines. SAE Technical Paper Series 820040, Feb. 1982. 
17. Vandromme, D.: Contribution to the Modeling and Prediction of Variable Density Flows. Ph.D. thesis presented at the University of Science and Technology, Lille, France, 1983.

18. Kline, S. J.; Cantwell, B. J.; and Lilley, G. M.: Proceeding of the 1980-81 AFOSR-HTTM Stanford Conference on Complex Turbulent Flow, Stanford University, Stanford, Calif., 1981.

19. Marvin, J. G.: Turbulence Modeling for Computational Aerodynamics. AIAA J., vol. 21, no. 21, July 1983, pp. 941-955.

20. Marvin, J. G.: Modeling of Turbulent Separated Flows for Aerodynamic Applications. Recent Advances in Aerodynamics, Springer-Verlag, ed. by A. Krothapalli and C. A. Smith, Proceedings of an International Symposium held at Stanford University, Aug. 22-26, 1983.

21. Delery, J.; and Marvin, J. G.: Shock-Wave Boundary Layer Interactions. AGARDograph No. 280, Feb. 1986.

22. van Driest, E. R.: Problem of Aerodynamic Heating: Aeronaut. Engin. Rev., vol. 15, no. 10, Oct. 1956, pp. 26-41.

23. Sandborn, V. A.: A Review of Turbulence Measurements in Compressible Flow. NASA TM X-62337, 1974.

24. Maise, G.; and McDonald, H.: Mixing Length and Kinematic Eddy Viscosity in a Compressible Boundary Layer. AIAA J., vol. 6, no. 1, Jan. 1968, pp. 73-79.

25. Hopkins, E. J.; and Inouye, M.: An Evaluation of Theories for Predicting Turbulent Skin Friction and Heat Transfer on Flat Plates at Supersonic and Hypersonic Mach Numbers. AIAA J., vol. 9, no. 6, Jun. 1971, pp. 993-1003.

26. Shang, J. S.: Computation of Hypersonic Turbulent Boundary Layers with Heat Transfer. AIAA J., vol. 12 , no. 7, July 1974 , pp. 883-884.

27. McDonald, H.: Mixing Length and Kinematic Eddy Viscosity in Low Reynolds Number Boundary Layer. Rep. J214453-1, Res. Lab., United Aircraft Corp., Sept. 1970.

28. Bushnell, D. M.; and Morris, D. J.: Shear-Stress, Eddy-Viscosity, and Mixing-Length Distributions in Hypersonic Turbulent Boundary Layers. NASA TM X-2310, 1971.

29. Bushnell, D. M.; Cary, A. M.; Jr. and Holley, B. B.: Mixing Length in Low Reynolds Number Compressible Turbulent Boundary Layers. AIAA TN, AIAA J., vol. 13, no. 8, Aug. 1975, pp. 1119-1121.

30. Bushnell, D. M.; Cary, A. M., Jr.; and Harris, J. E.: Calculation Methods for Compressible Turbulent Boundary Layers-1976. NASA SP-422, 1977.

31. Dash, S.; Weilerstein, G.; and Vaglio-Laurin, R.: Compressibility Effects in Free Turbulent Shear Flows. AFOSR TR-75-436, Aug. 1975. 
32. Chinzie, N.; Masuya, G.; Komuro, T.; Murakami, A.; and Kudou, K.: Spreading of Two-Stream Supersonic Mixing Layer. Phys. Fluids, vol. 29, no. 5, May 1986. 


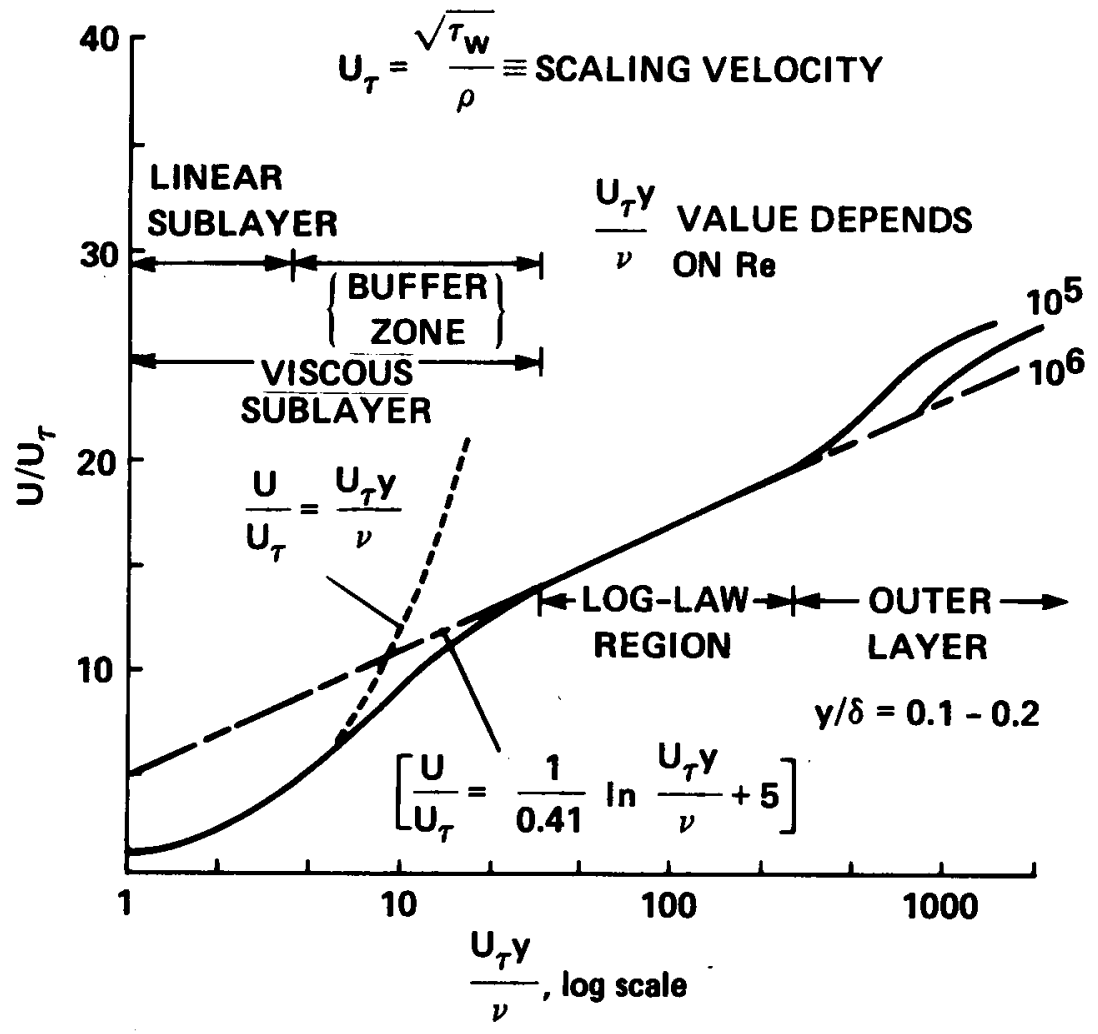

Fig. 1. Composite sketch of a turbulent boundary layer.

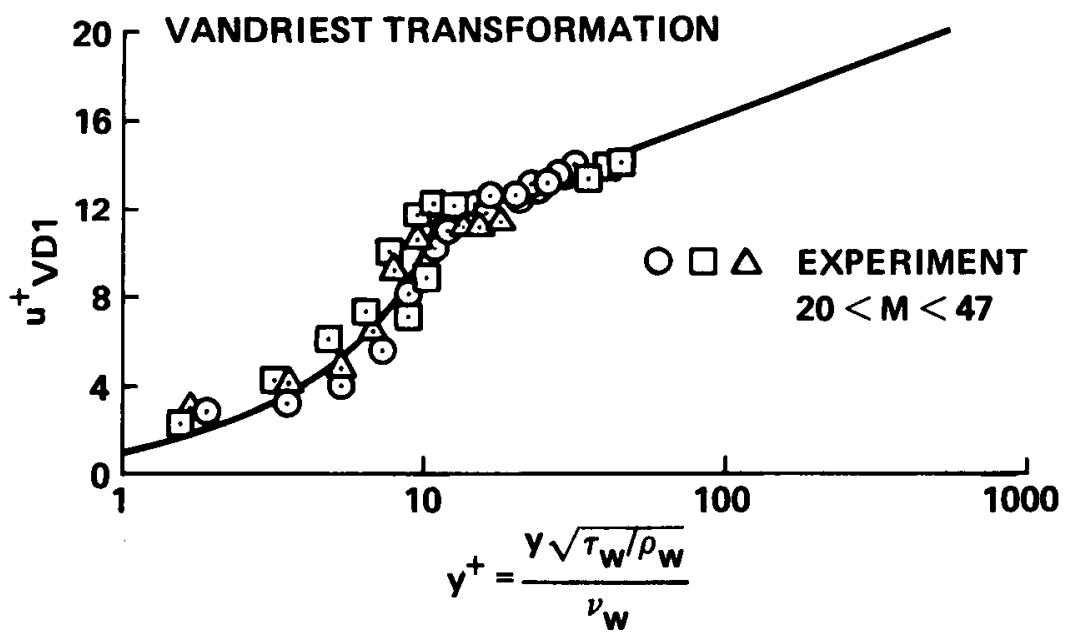

Fig. 2. A log-law representation of hypersonic boundary layer profiles. 


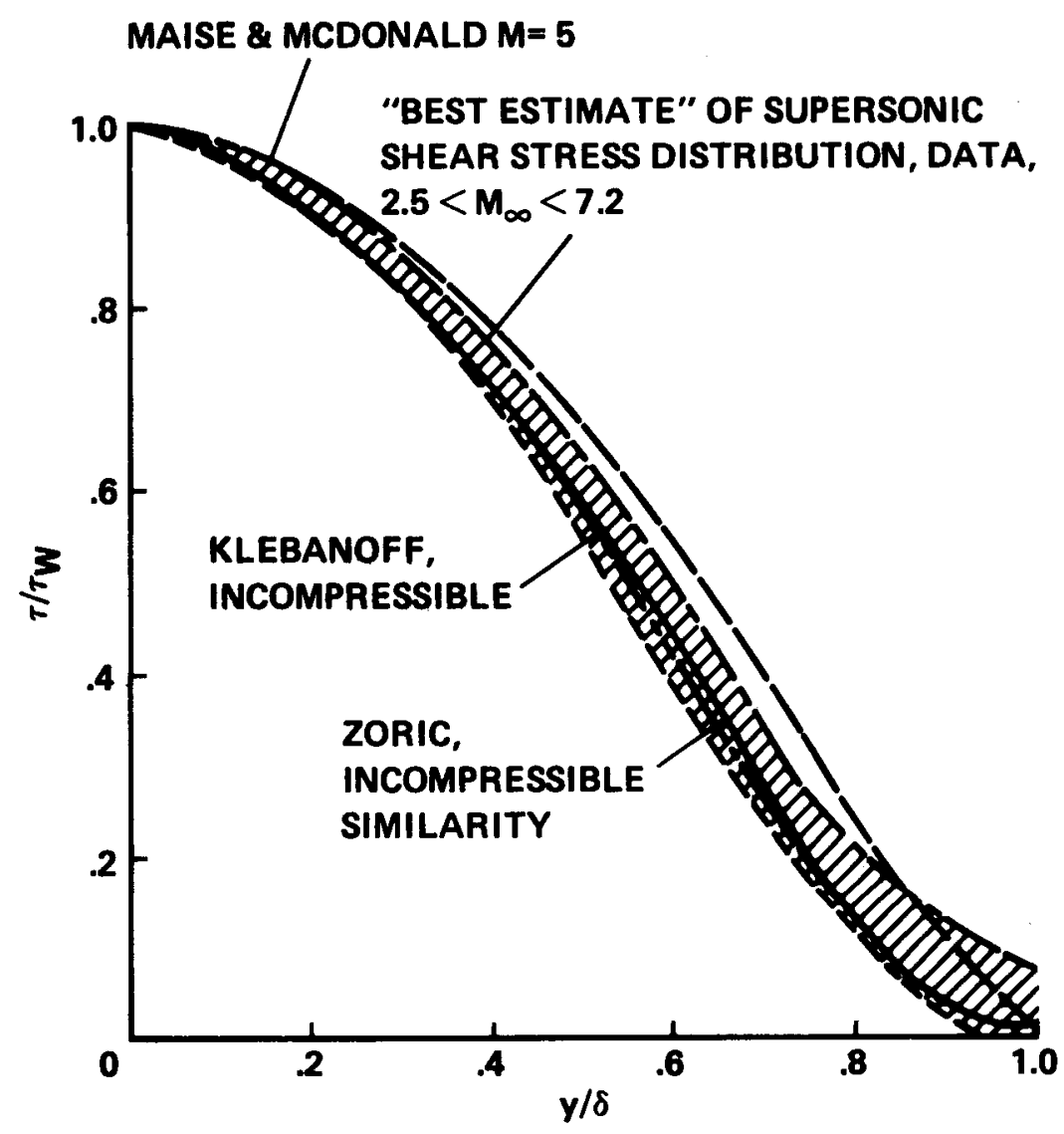

Fig. 3. A comparison of the best estimate of shear stress data with incompressible measurements and with Maise and McDonald's compressible approximation. 


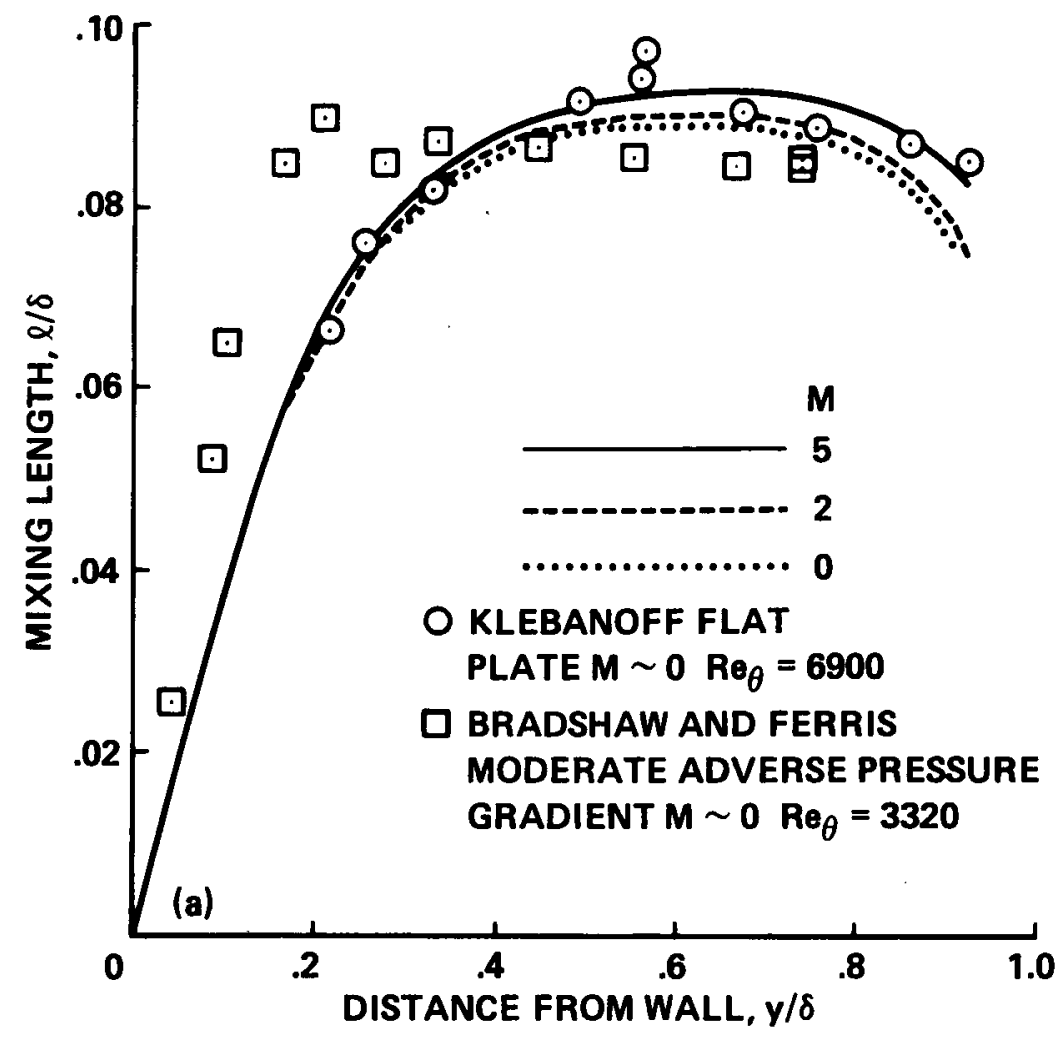

(a) Mixing length.

Fig. 4. Mixing length and scaled eddy viscosity from Maise and McDonald. 


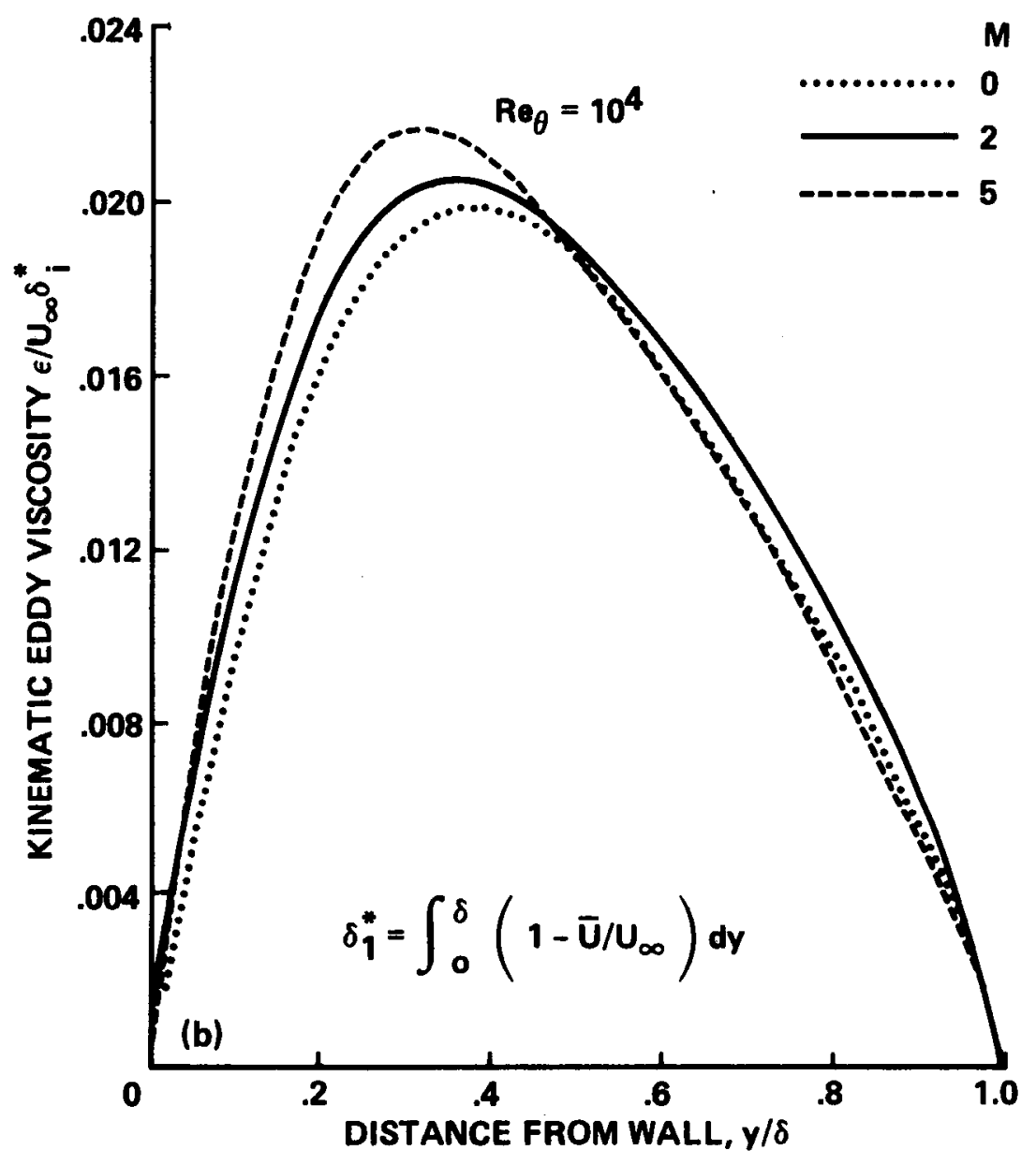

(b) Scaled eddy viscosity.

Fig. 4. Concluded. 


\section{FLAT PLATE SKIN FRICTION}
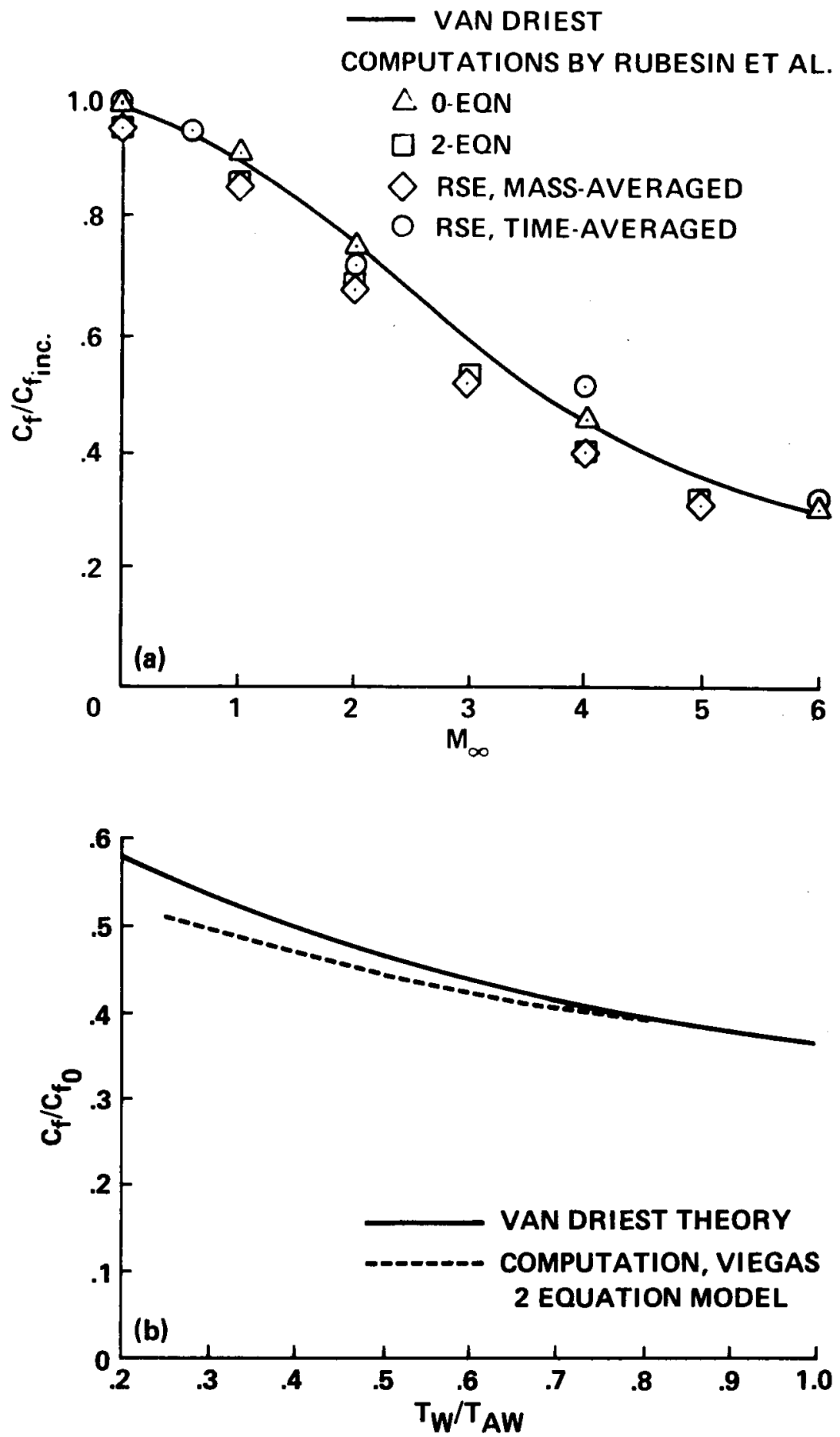

Fig. 5. Ability of turbulence models to predict compressibility effects: (a) adiabatic wall temperature; (b) Mach 5 and variable wall temperature. 
FLAT PLATE FLOW

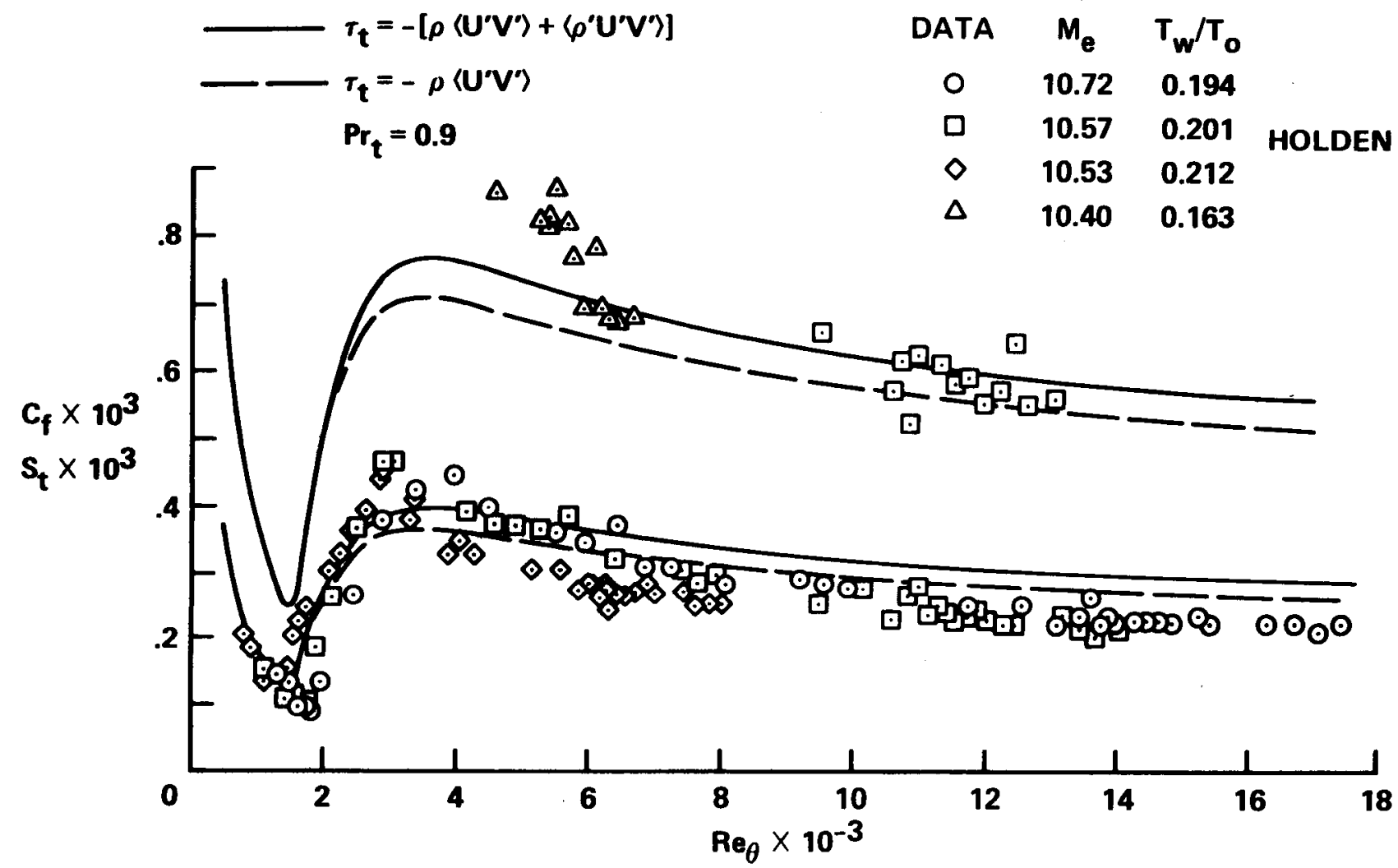

Fig. 6. A comparison of experimental skin friction and heat transfer with computation using a turbulence model with and without corrections for density fluctuations. 


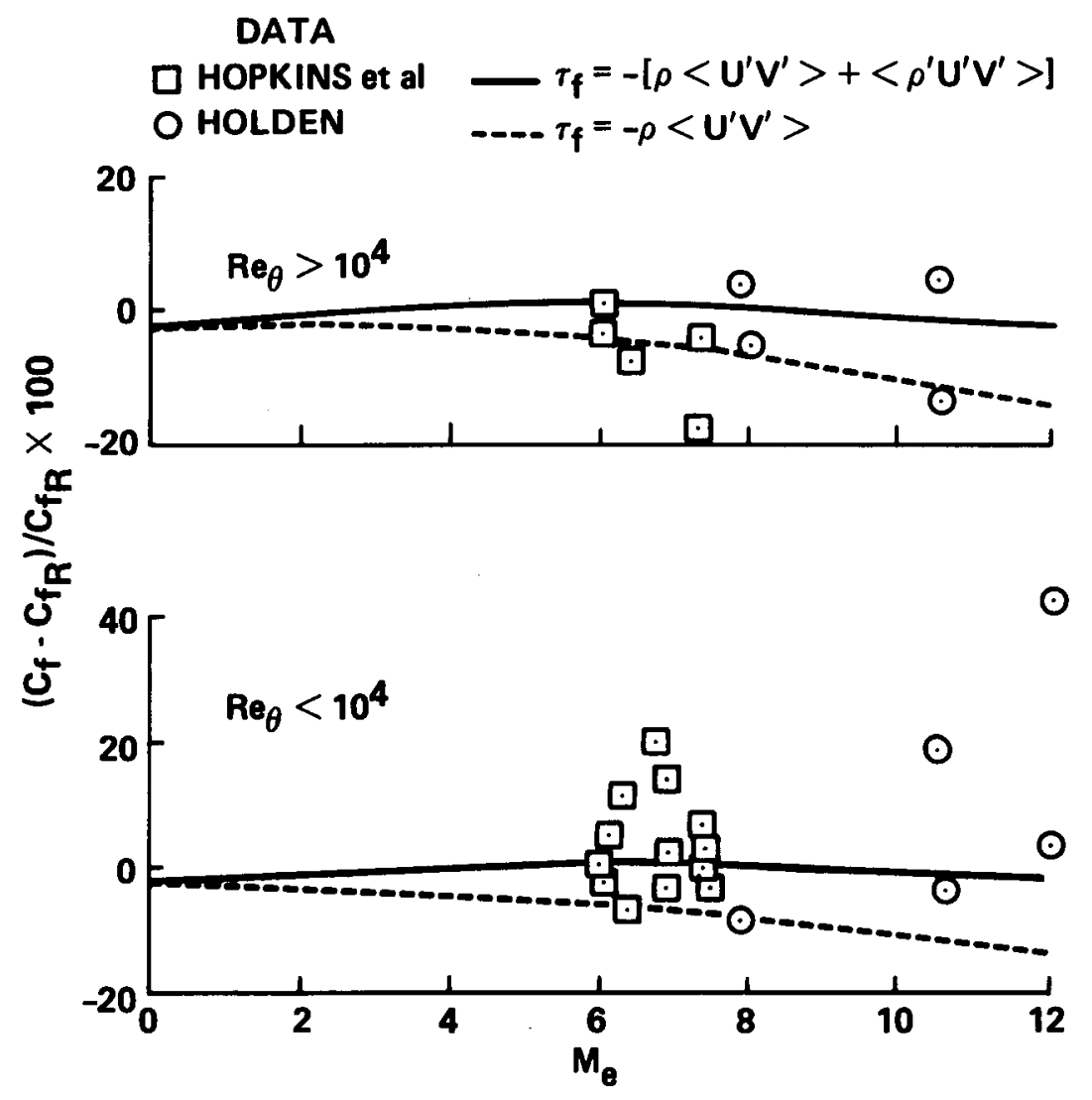

Fig. 7. Error in skin friction prediction for two ranges of Reynolds numbers using a turbulence model with and without correction for density fluctuations. 


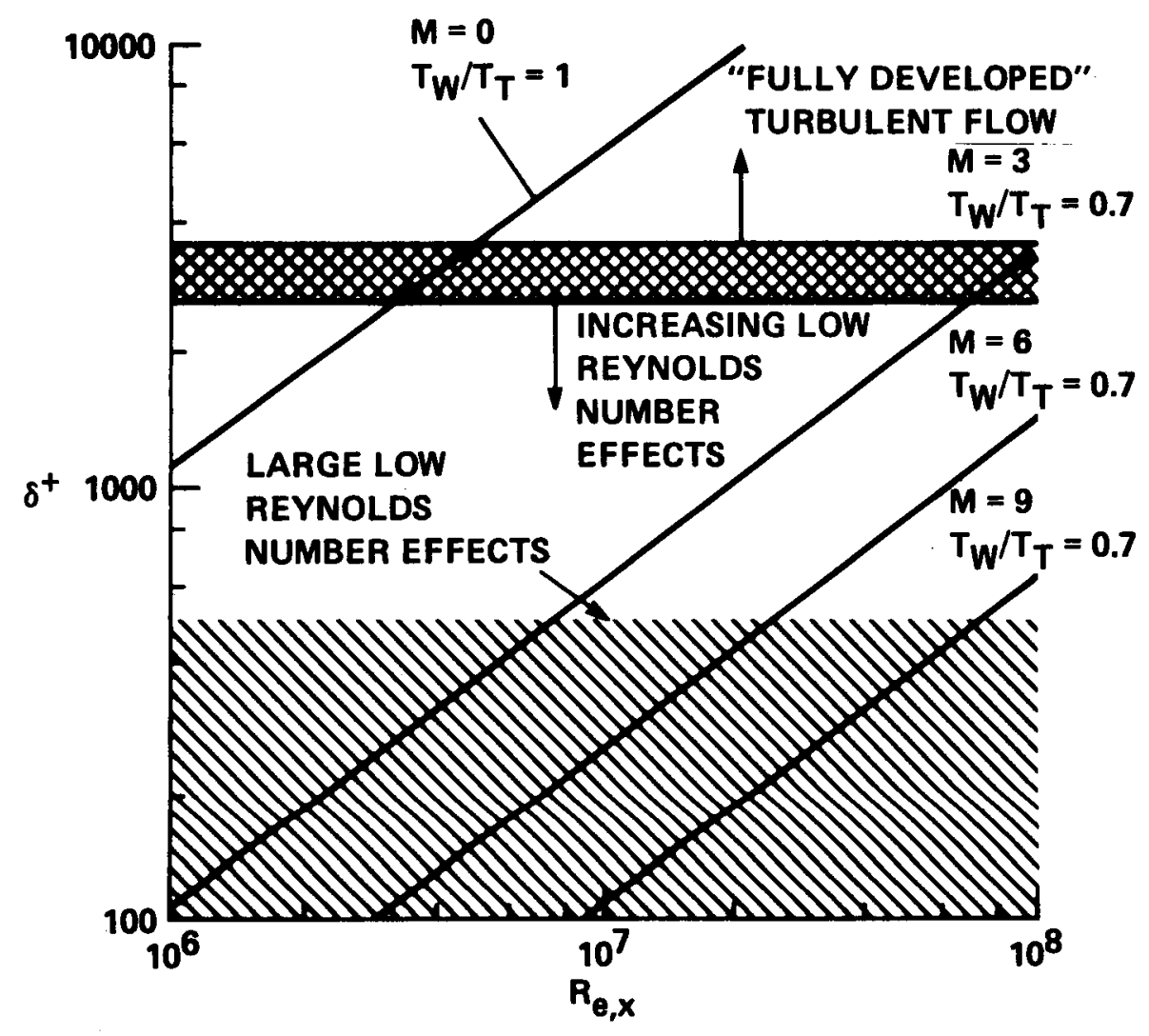

Fig. 8. Increasing importance of Low Reynolds number effects with Mach number. 
2-D ATTACHED - PRESSURE GRADIENT

\begin{tabular}{|c|c|c|c|c|c|}
\hline REFERENCE & $\begin{array}{l}\text { EXPERIMENTAL } \\
\text { CONFIGURATION }\end{array}$ & $M_{\infty}$ & $\operatorname{Re}_{\theta_{\infty}} \times 10^{-4}$ & $T_{w} / T_{0}$ & $\mathrm{P}_{\text {max }}^{+}$ \\
\hline ZWARTS & 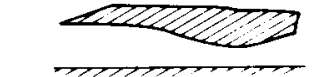 & 4.02 & 3.5 & 1 & 0.004 \\
\hline $\begin{array}{l}\text { PEAKE, BRAKMANN } \\
\text { AND ROMESKIE }\end{array}$ & & 3.93 & 1.1 & 1 & 0.006 \\
\hline $\begin{array}{l}\text { STUREK AND } \\
\text { DANBERG }\end{array}$ & & 3.54 & $2.0-2.8$ & 1 & $\begin{array}{l}0.0085 \\
0.0125\end{array}$ \\
\hline $\begin{array}{l}\text { LEWIS, GRAN } \\
\text { AND KUBOTA }\end{array}$ & $E_{2}$ & 3.98 & 0.5 & 1 & 0.011 \\
\hline $\begin{array}{l}\text { KUSSOY AND } \\
\text { HORSTMAN }\end{array}$ & $\begin{array}{l}c \rightarrow \\
4 b\end{array}$ & 6.7 & 0.8 & 0.43 & 0.07 \\
\hline $\begin{array}{l}\text { KUSSOY AND } \\
\text { HORSTMAN }\end{array}$ & +2 & 2.3 & $104-2270$ & 1 & 0.12 \\
\hline
\end{tabular}

Fig. 9. Benchmark flows with pressure gradient effects. 


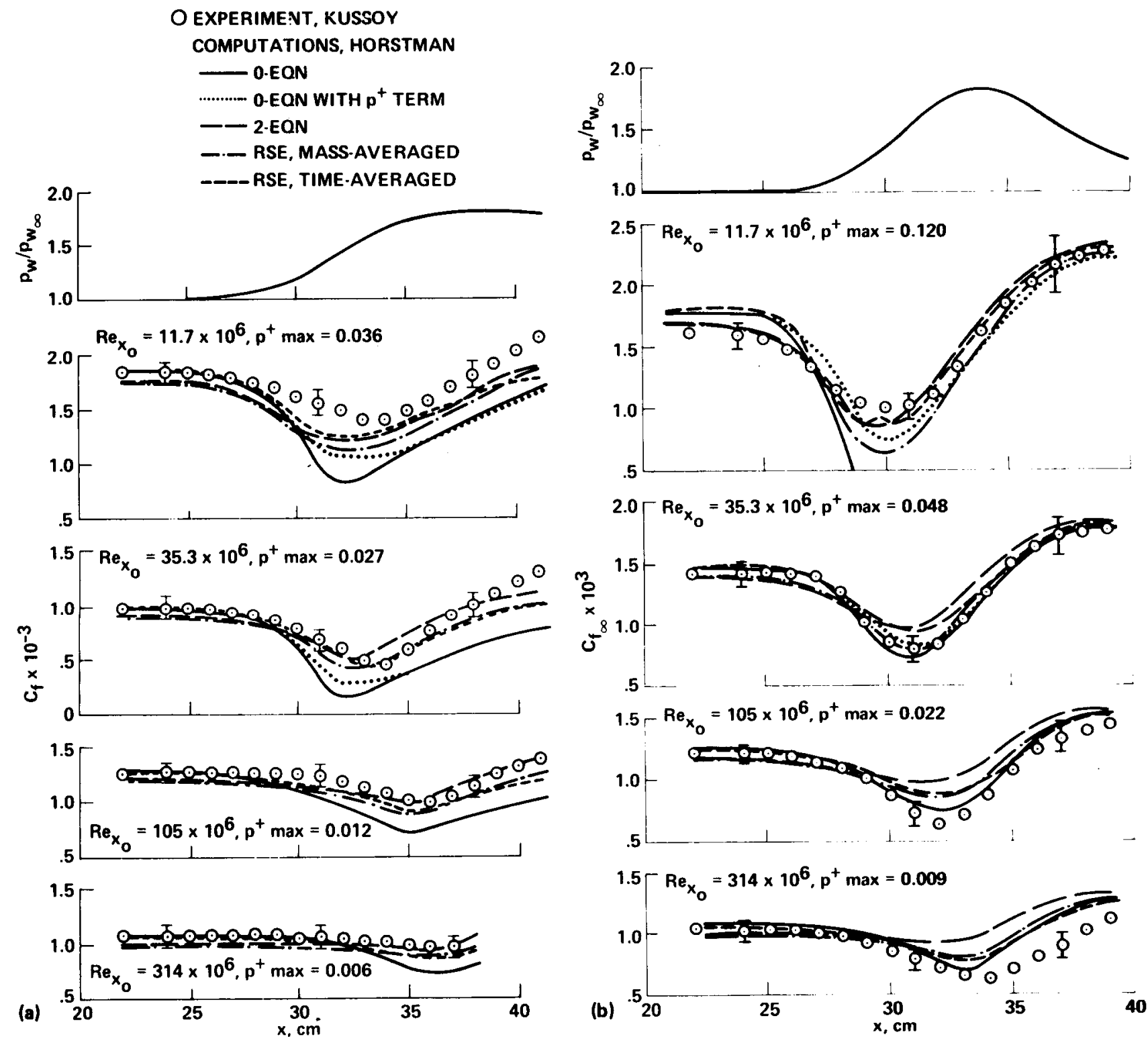

Fig. 10. A comparison of computations using various models with experiment. 


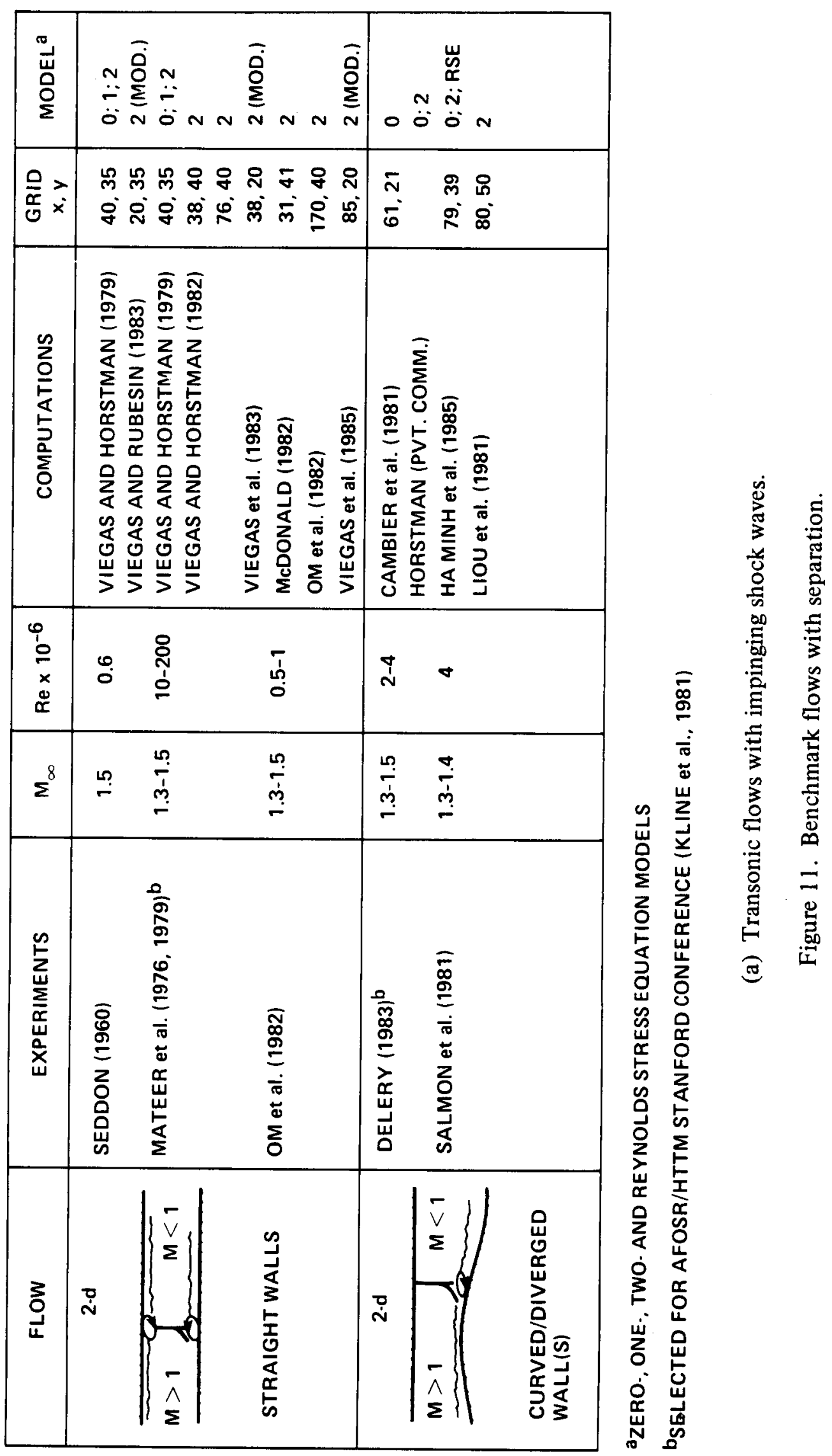




\begin{tabular}{|c|c|c|c|}
\hline 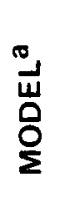 & 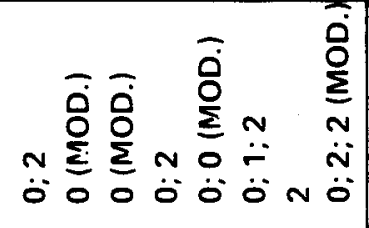 & $0 \stackrel{N}{0}$ & $\begin{array}{l}\overline{\dot{0}} \\
\frac{0}{3}\end{array}$ \\
\hline 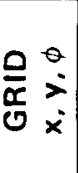 & 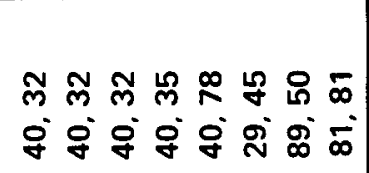 & 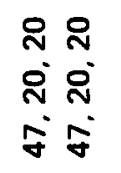 & $\begin{array}{l}\text { D } \\
\text { ì } \\
\text { \&े }\end{array}$ \\
\hline $\begin{array}{l}\frac{n}{2} \\
0 \\
\frac{1}{5} \\
5 \\
5 \\
\frac{a}{2} \\
0 \\
0\end{array}$ & 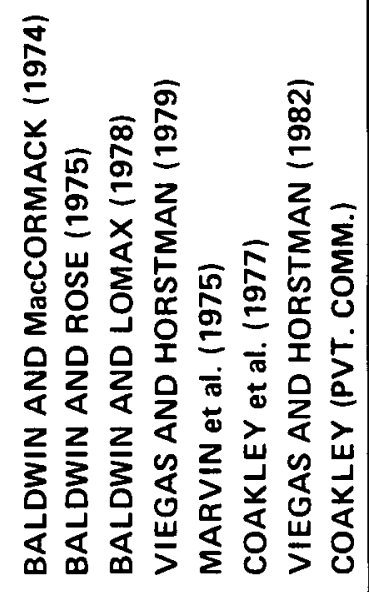 & 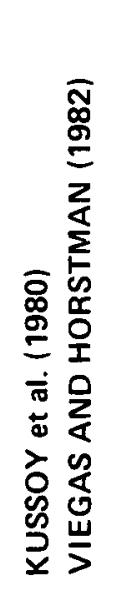 & 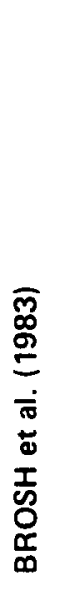 \\
\hline $\begin{array}{l}0 \\
1 \\
0 \\
x \\
\ddot{x}\end{array}$ & $\approx 5$ & $\stackrel{m}{m}$ & $\stackrel{\infty}{ }$ \\
\hline$\Sigma^{8}$ & $\stackrel{\infty}{\infty} m \quad \stackrel{N}{\sim}$ & N & $m$ \\
\hline 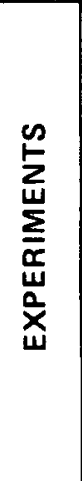 & 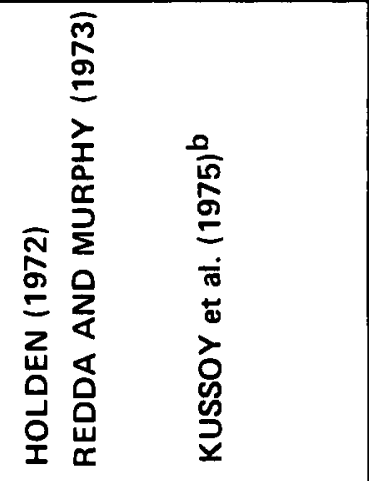 & 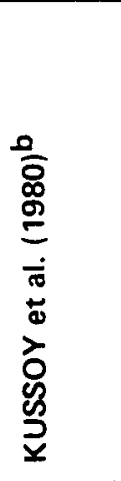 & 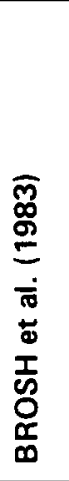 \\
\hline క్ & 11 & mं & \\
\hline
\end{tabular}

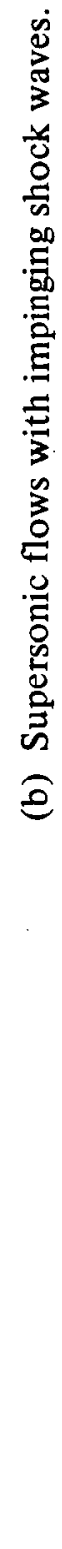




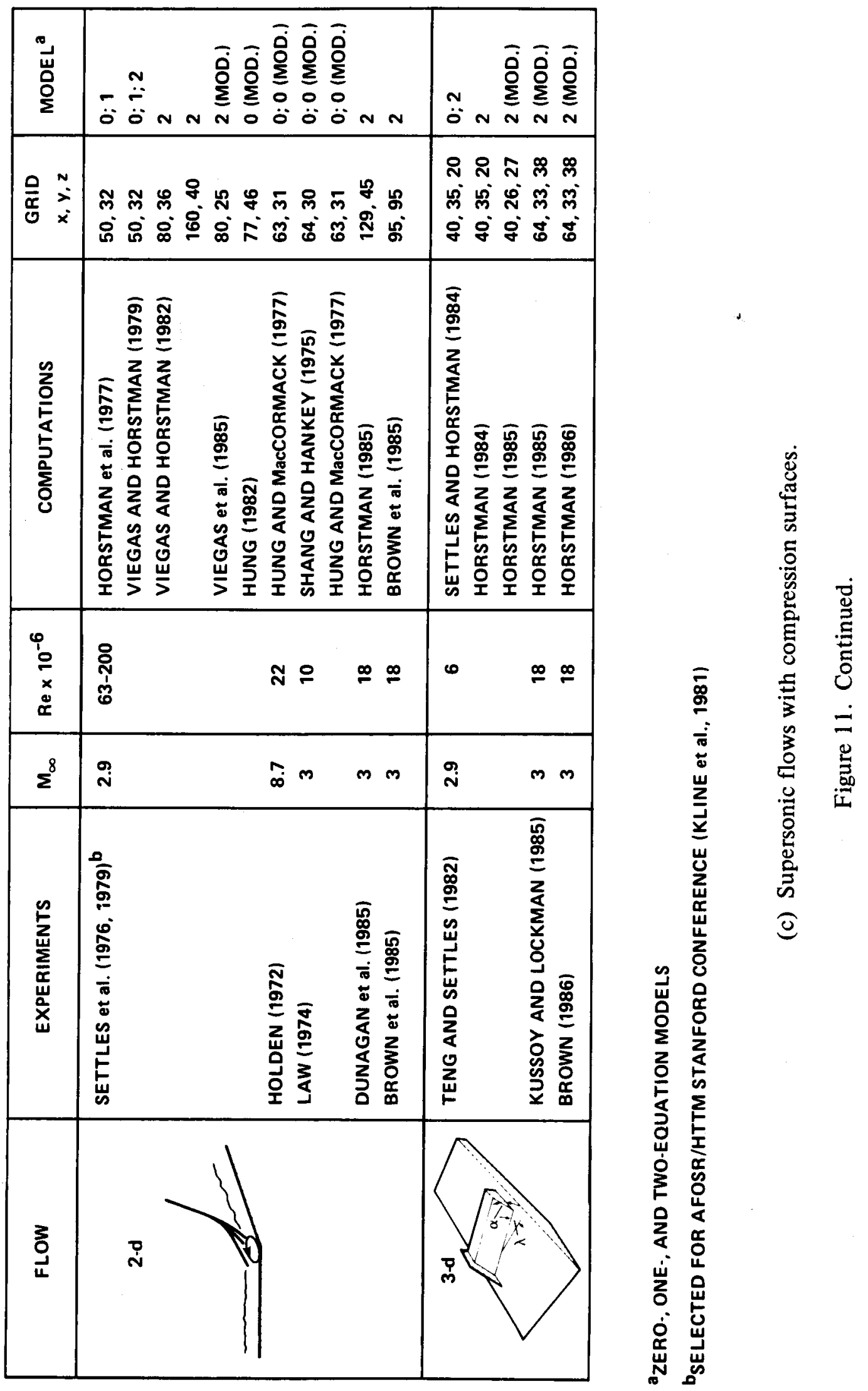




\begin{tabular}{|c|c|c|}
\hline $\begin{array}{l}\stackrel{0}{u} \\
\text { Oे } \\
\stackrel{0}{\Sigma}\end{array}$ & 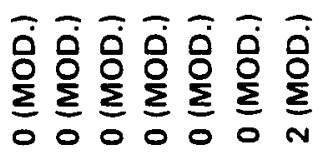 & $\frac{\overline{0}}{\sum_{0}^{\circ}}$ \\
\hline 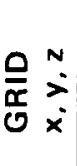 & 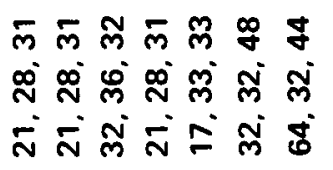 & $\begin{array}{l}\text { m } \\
\text { लें } \\
\tilde{q}\end{array}$ \\
\hline $\begin{array}{l}\text { D } \\
0 \\
0 \\
5 \\
5 \\
5 \\
0 \\
0 \\
0 \\
0\end{array}$ & 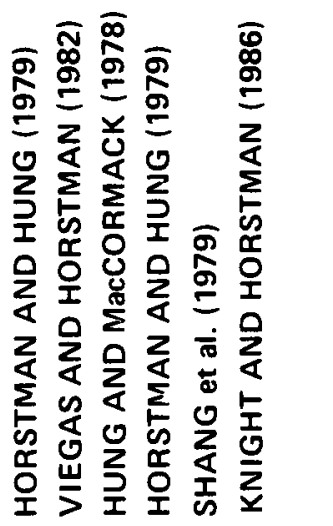 & 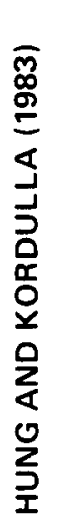 \\
\hline $\begin{array}{l}0 \\
\stackrel{0}{0} \\
x \\
\ddot{x}\end{array}$ & 잉 $\quad$ in $\stackrel{\infty}{0}$ ? & $\stackrel{\infty}{\circ}$ \\
\hline$\Sigma^{8}$ & $\sim \quad m m m$ & $m$ \\
\hline 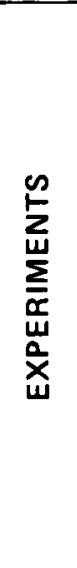 & 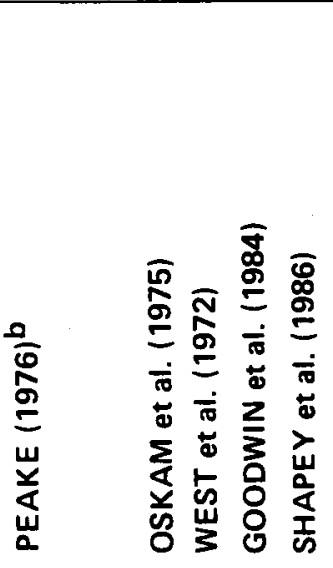 & 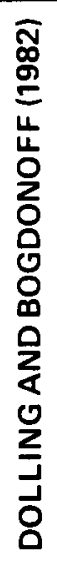 \\
\hline 疋 & m் & लं \\
\hline
\end{tabular}

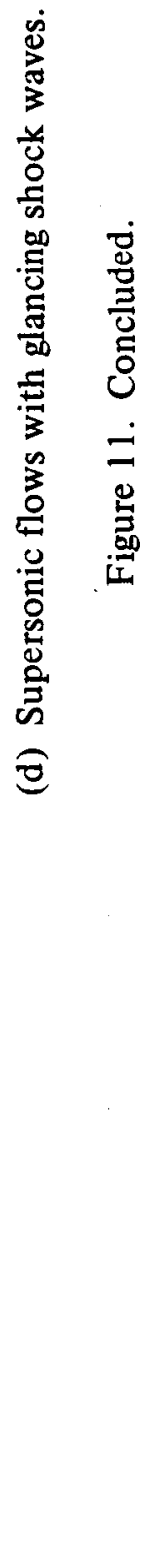




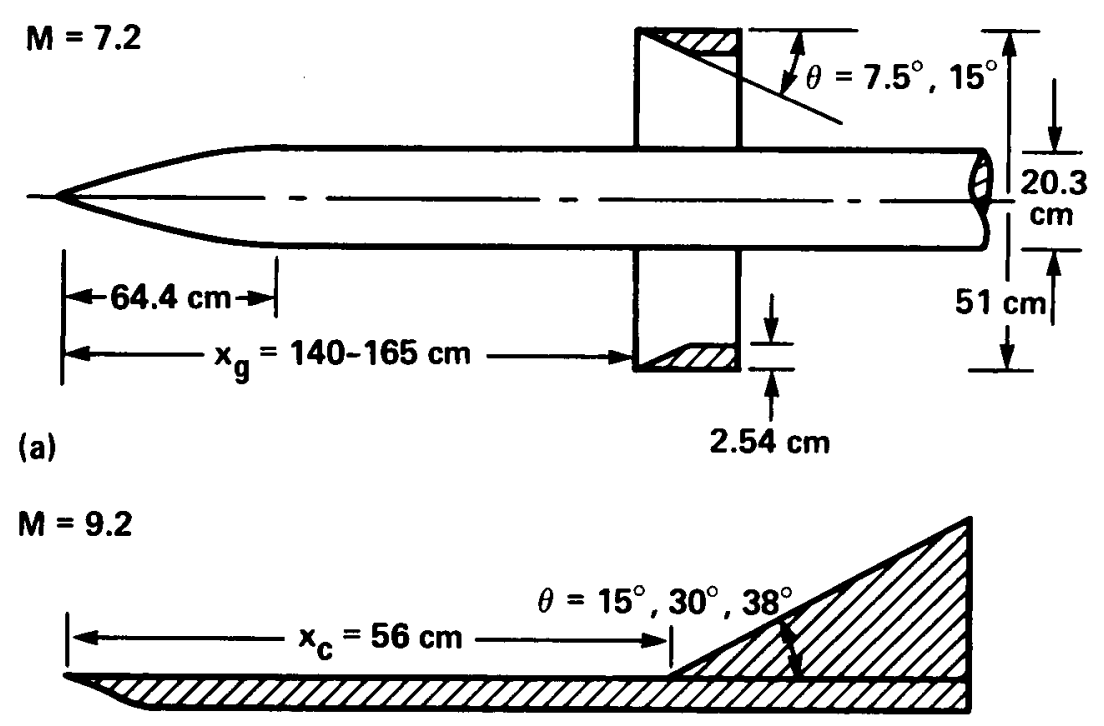

(b)

Fig. 12. Experimental arrangements for two flows used to assess models with compressibility corrections. 

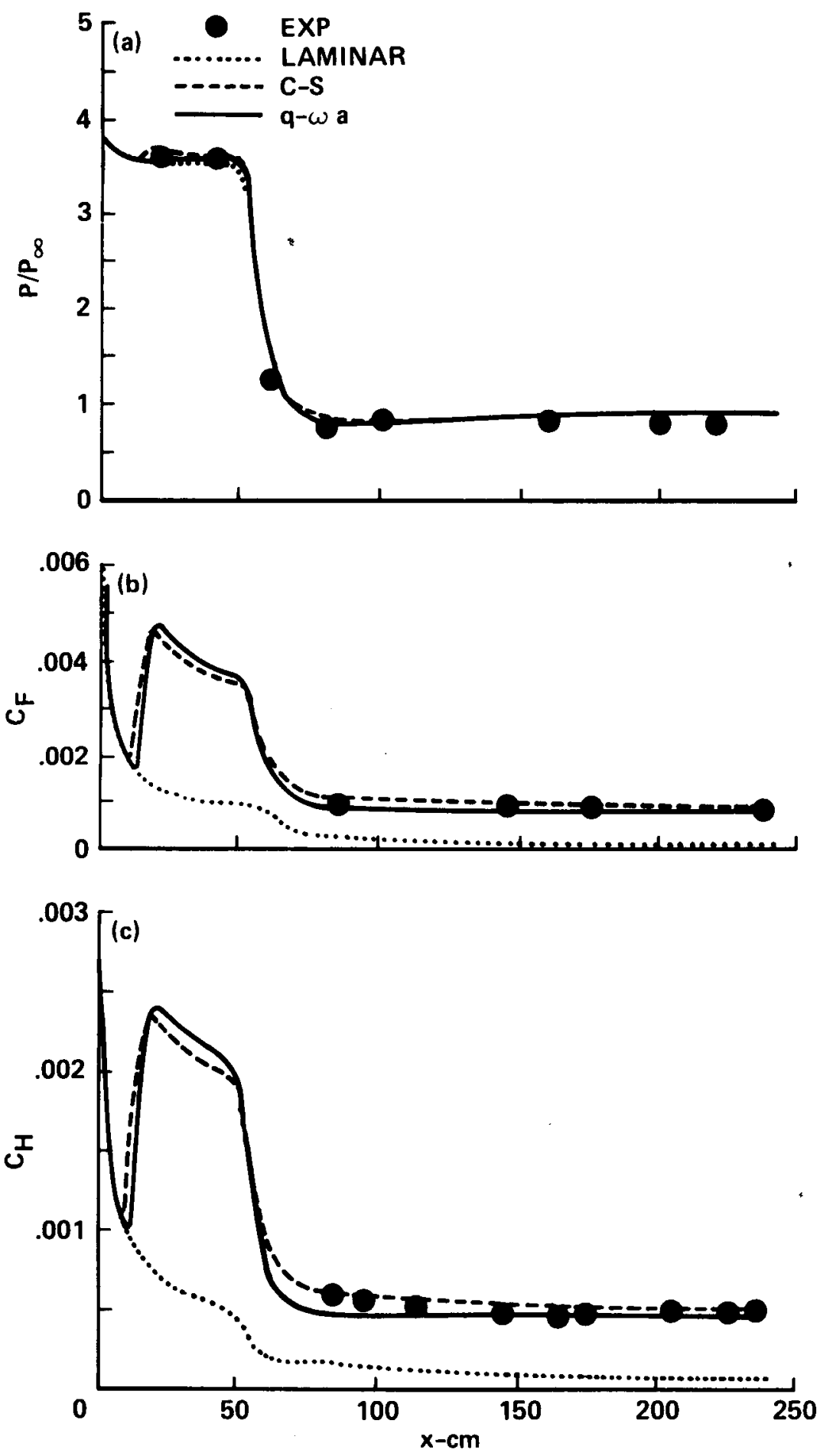

Fig. 13. Ogive-cylinder flow without the shock generator ring: (a) surface pressure; (b) skin friction; (c) surface heat transfer. 

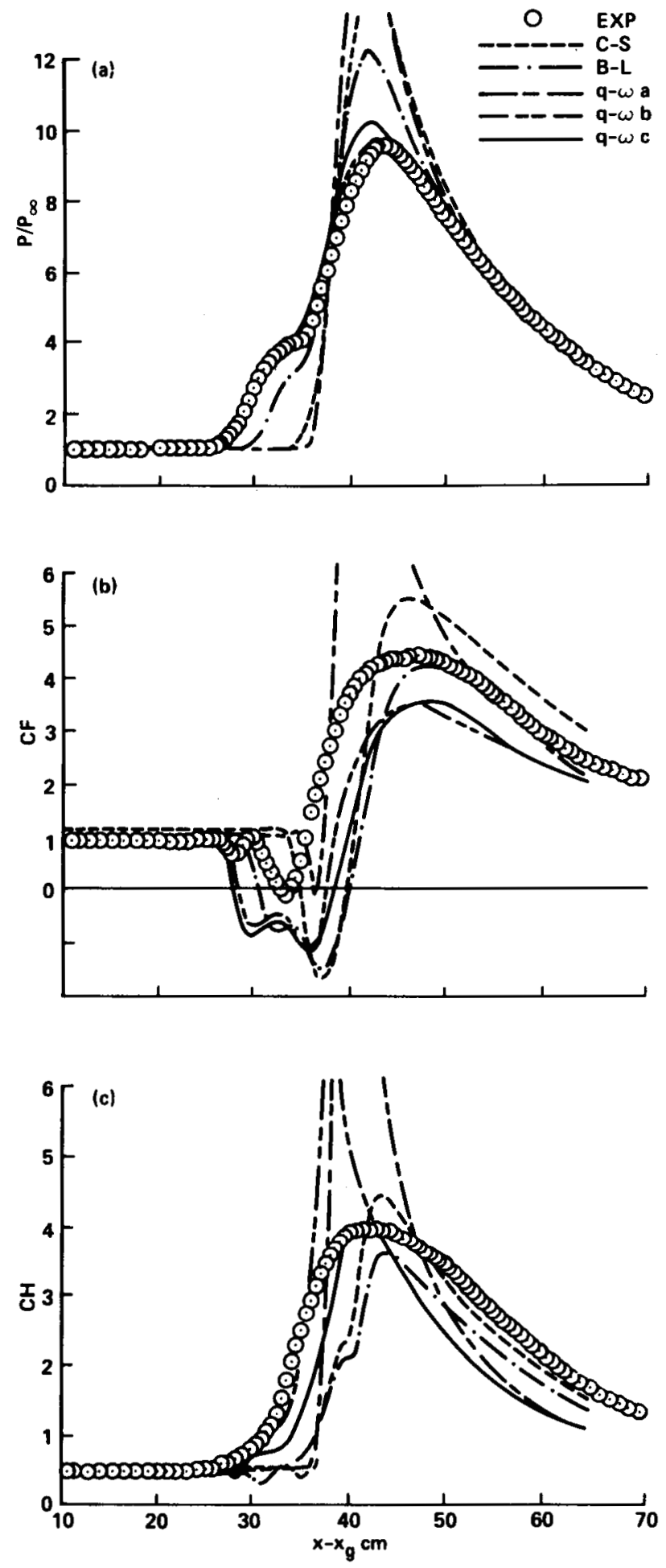

Fig. 14. Ogive-cylinder flow with $15^{0}$ shock-generator ring: (a) surface pressure; (b) skin friction; (c) surface heat transfer. 


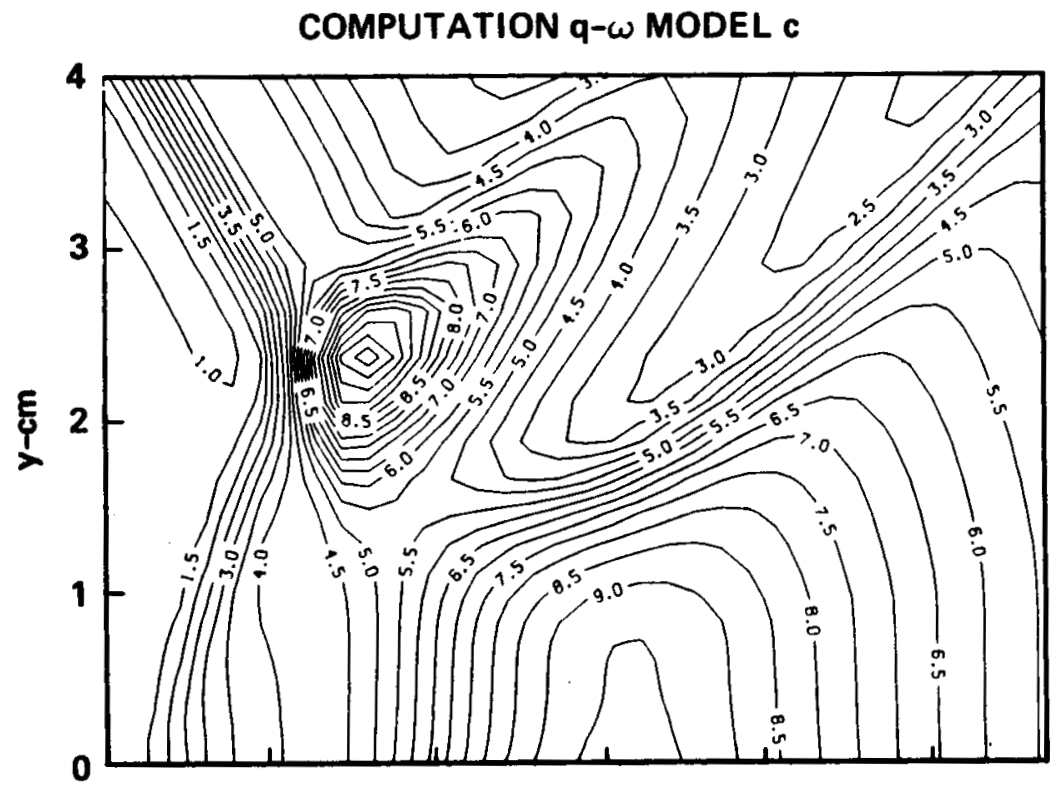

(a)

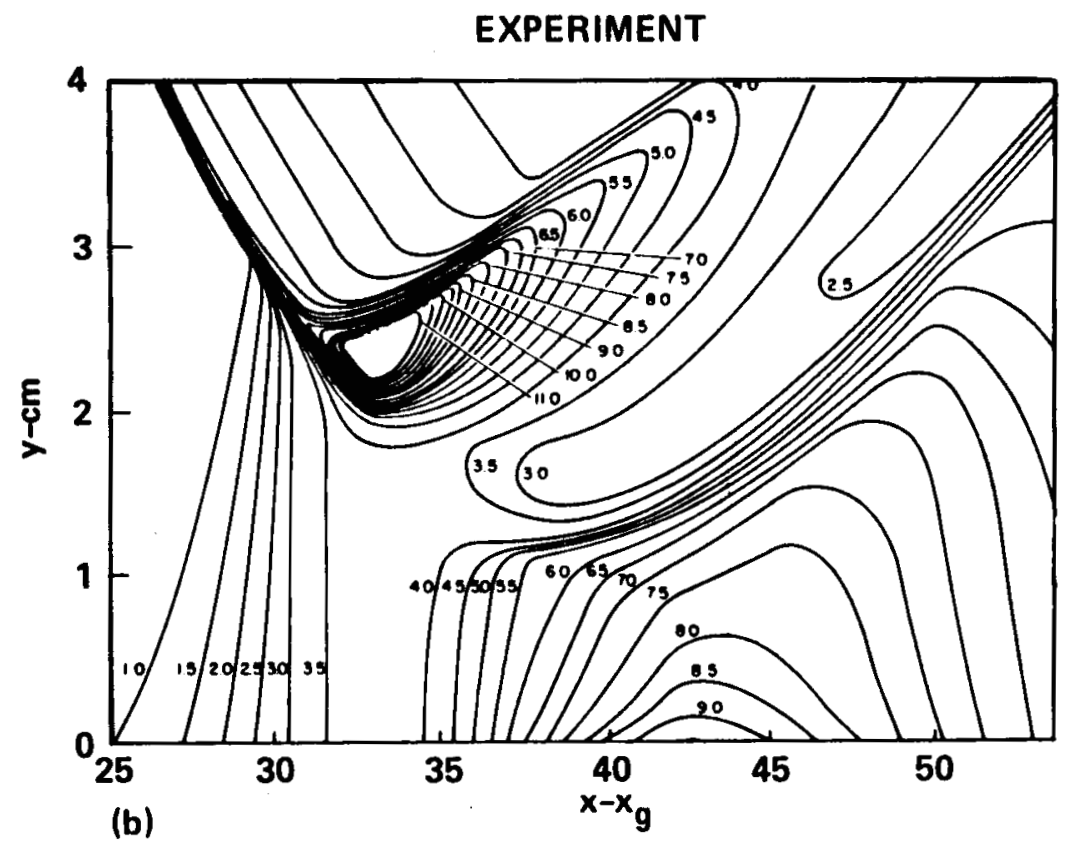

Fig. 15. Ogive-cylinder flow with $15^{\circ}$ shock generator ring: (a) computed pressure contours; (b) experimental pressure contours. 

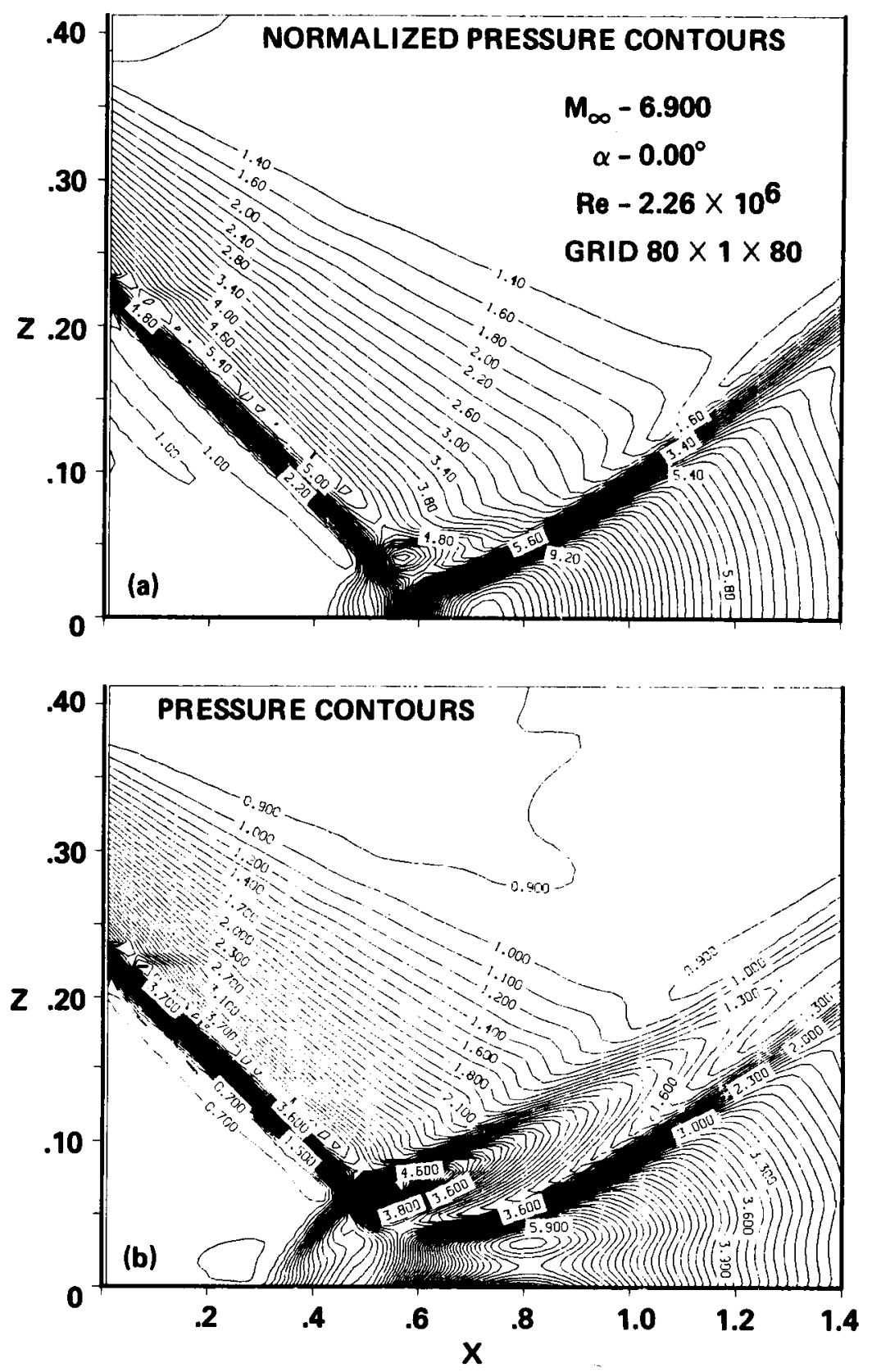

Fig. 16. Ogive-cylinder flow with $15^{0}$ shock generator ring: (a) zero-equation Baldwin-Lomax model; (b) $q-\omega$ model c 

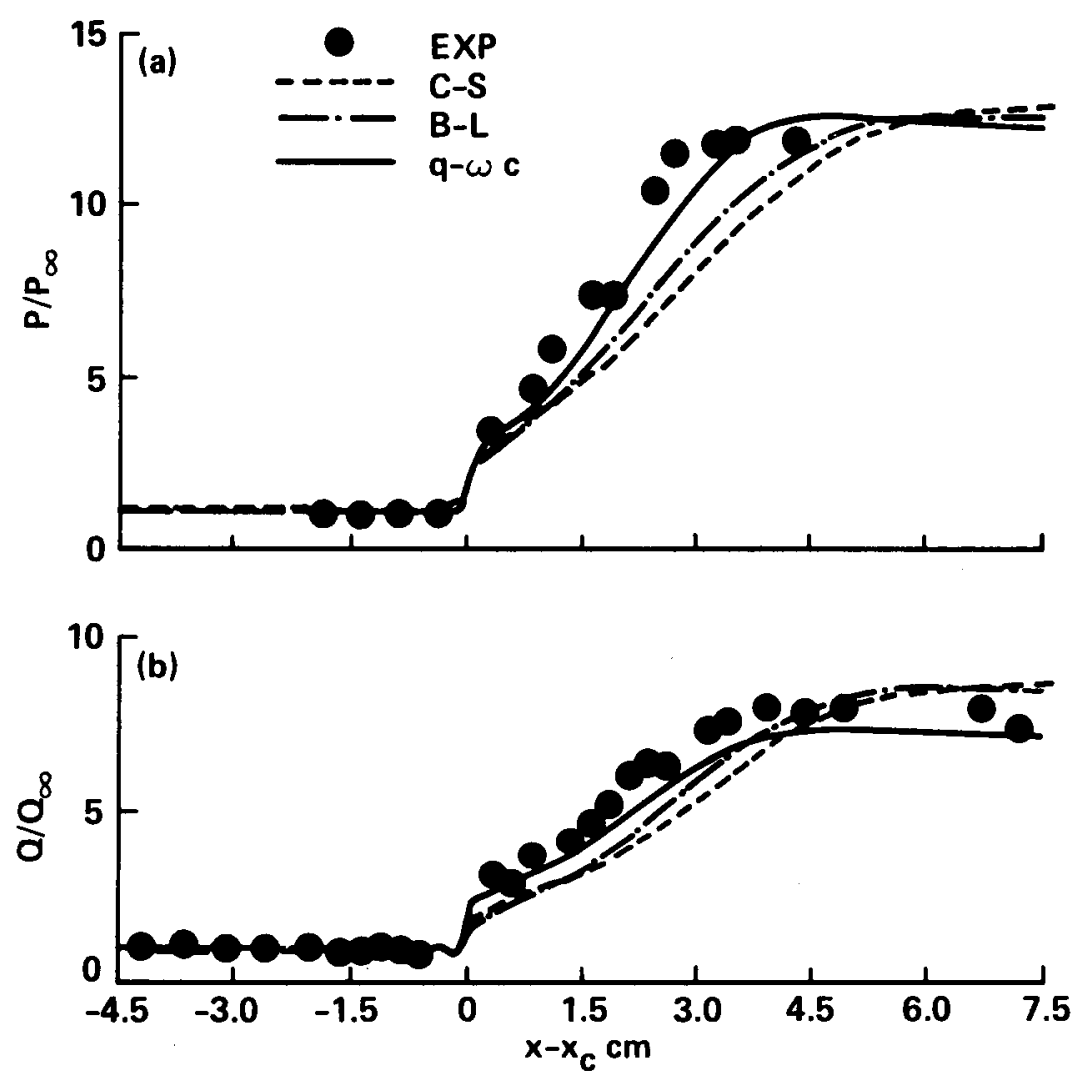

Fig. 17. Compression Corner Flow , $15^{\circ}$ corner angle: (a) surface pressure; (b) surface heat transfer. 

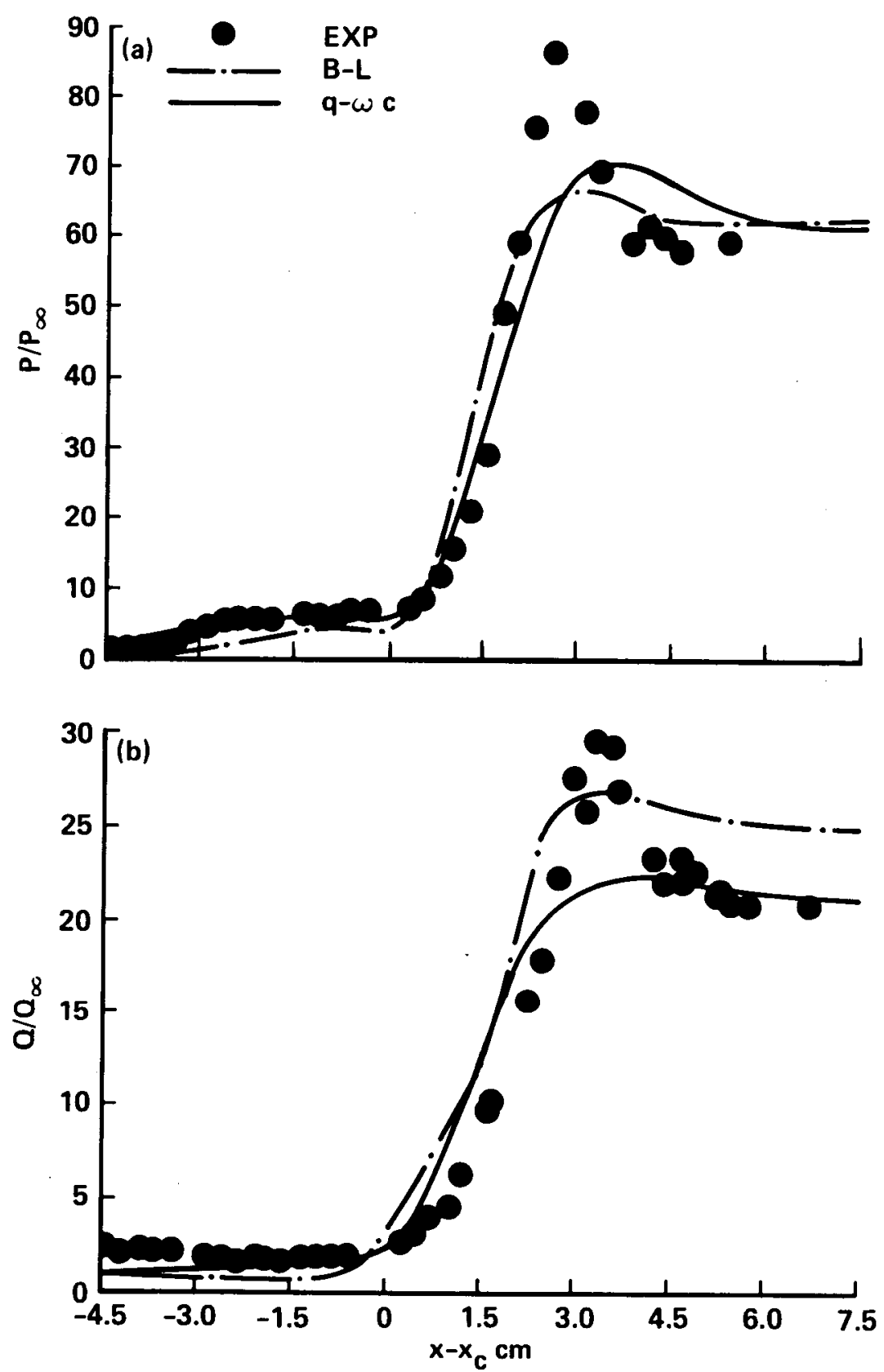

Fig. 18. Compression Corner Flow, $38^{0}$ corner angle: (a) surface pressure; (b) surface heat transfer. 
EXPERIMENT: SETTLES et al.

$$
\begin{array}{ll}
M_{\infty} \cong 2.85 & \operatorname{Re}_{\delta_{0}} \approx 1.6 \times 10^{6} \\
T_{w} / T_{T} \approx 1 & \delta_{0} \approx 2.5 \mathrm{~cm} \\
\alpha=16,20 \& 24^{\circ} &
\end{array}
$$

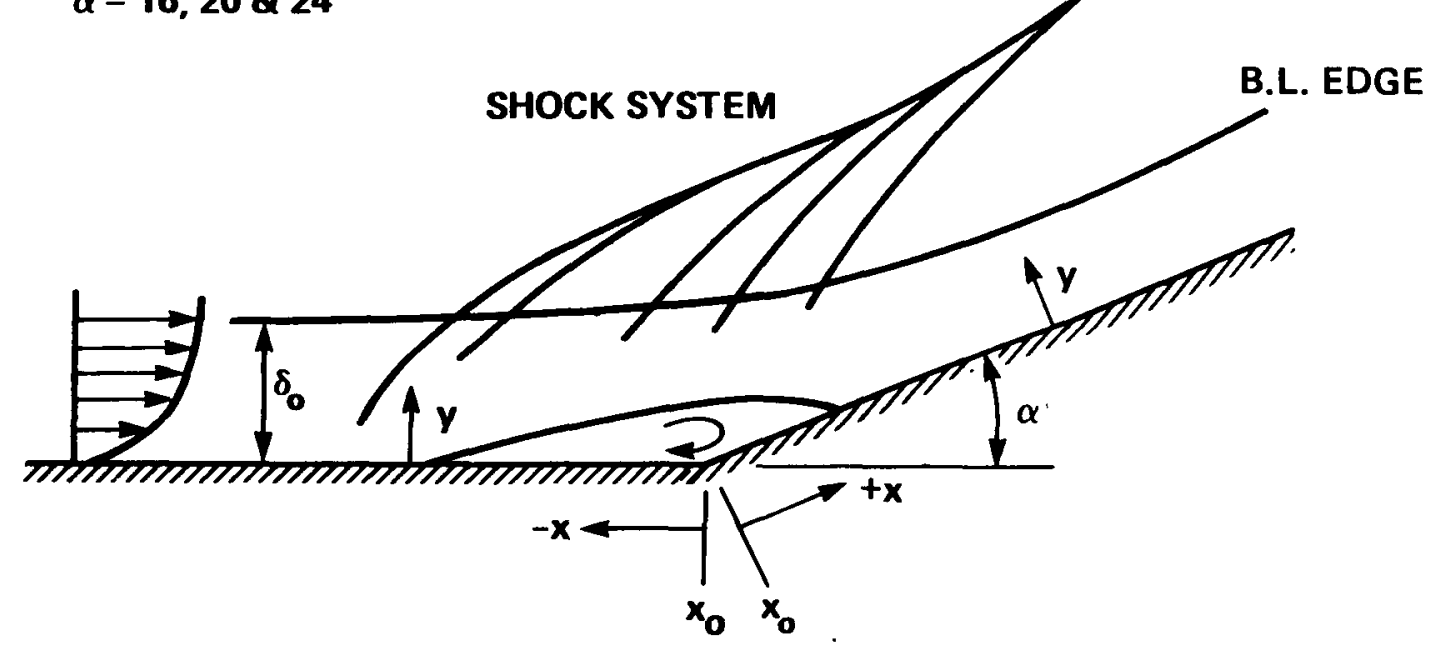

Fig. 19. Geometry and conditions of a compression corner experiment. 
TURBULENCE MODEL COMPARISON WITH WALL FUNCTIONS AND WITH INTEGRATION TO WALL

$20^{\circ}$ RAMP ANGLE
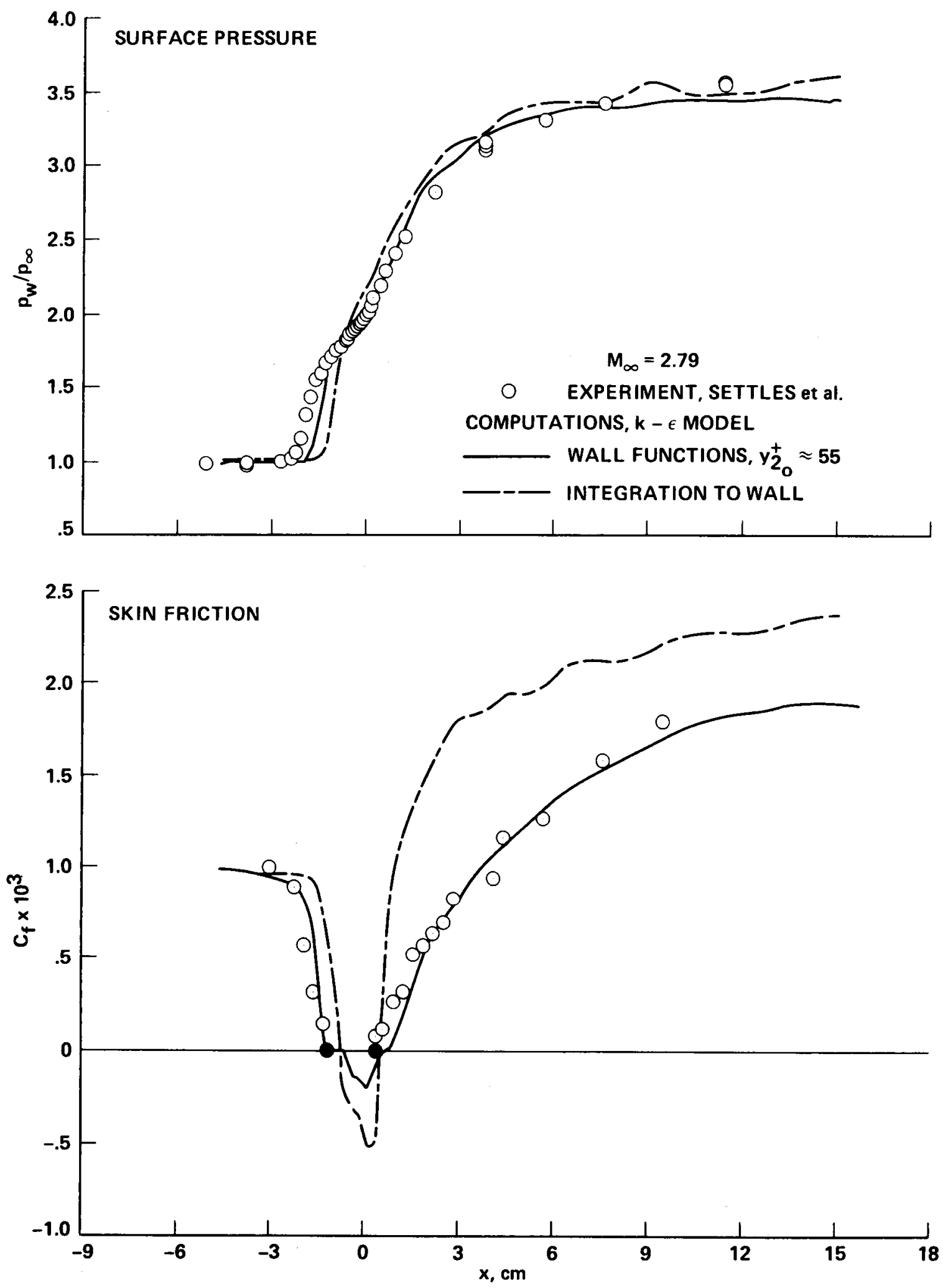

Fig. 20. Comparison of computations using $k-\epsilon$ model with experiment. 

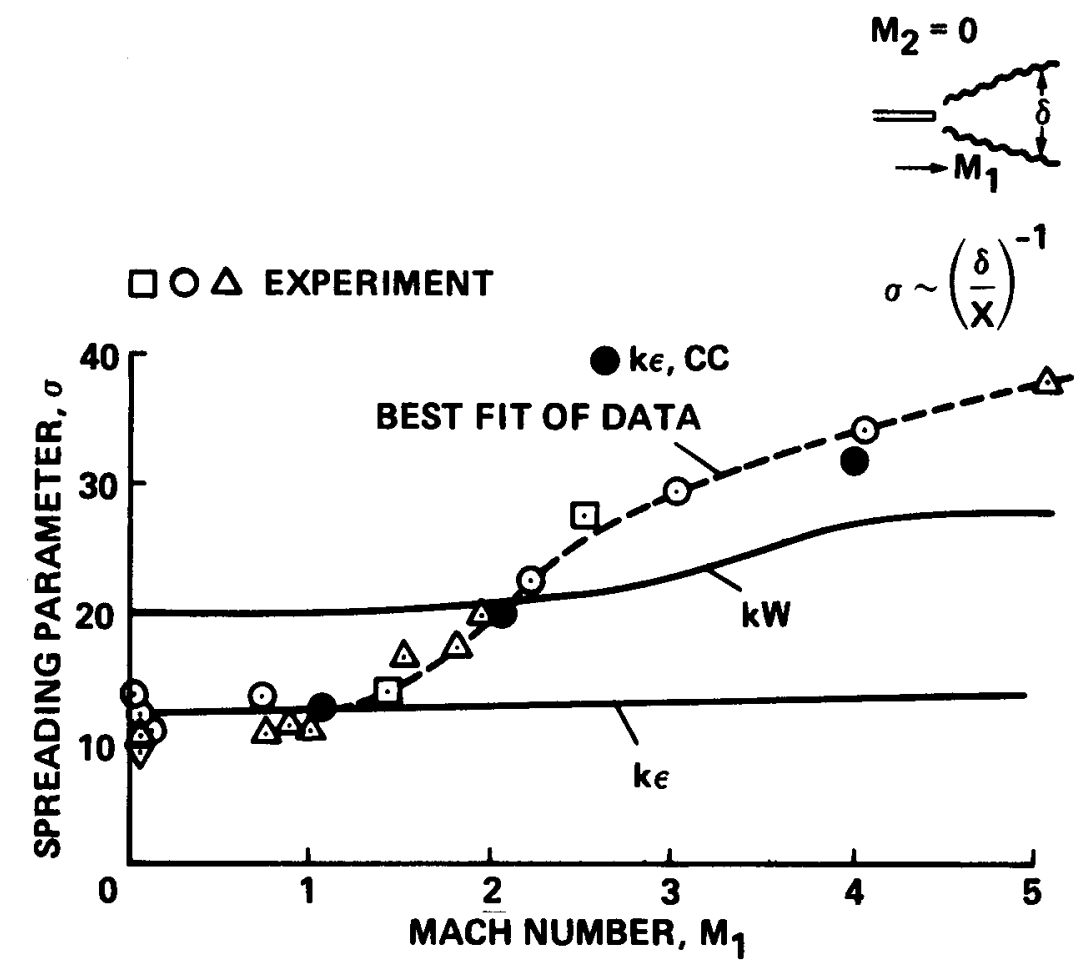

Fig. 21. Spreading rates for compressible 2-D shear layers. Data fits from NASA SP-321, 1972.

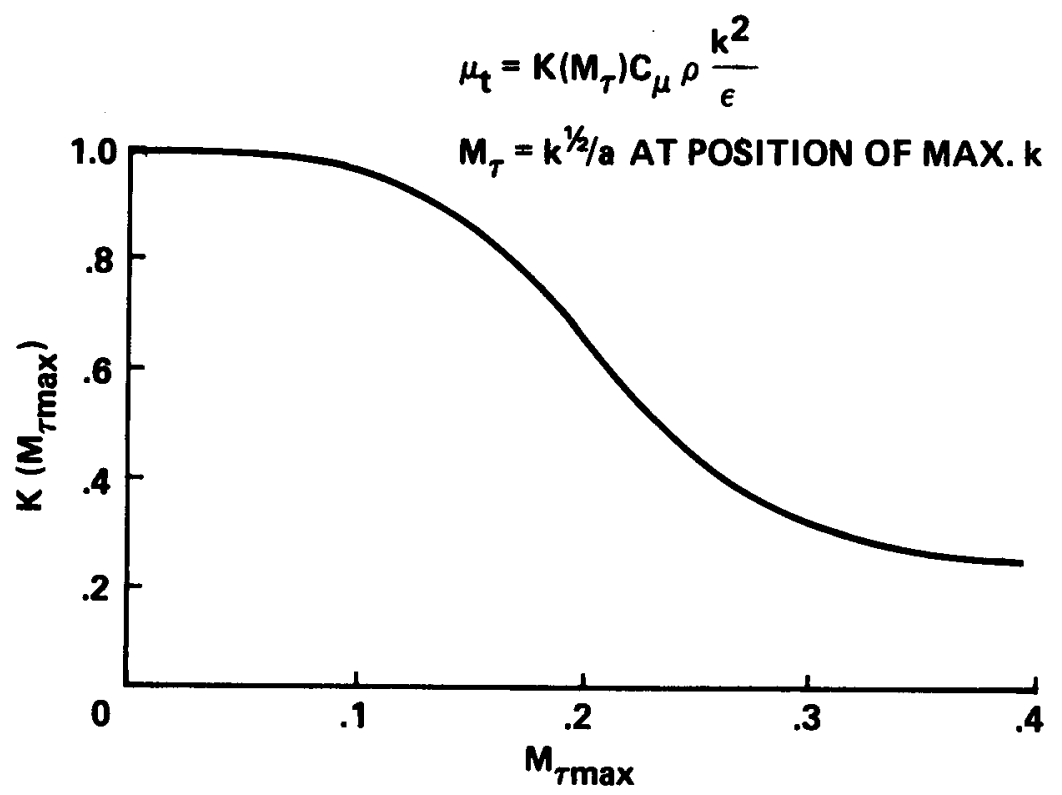

Fig. 22. Compressibility correction for $k-\epsilon$ model proposed by Dash, et. al ${ }^{31}$. 


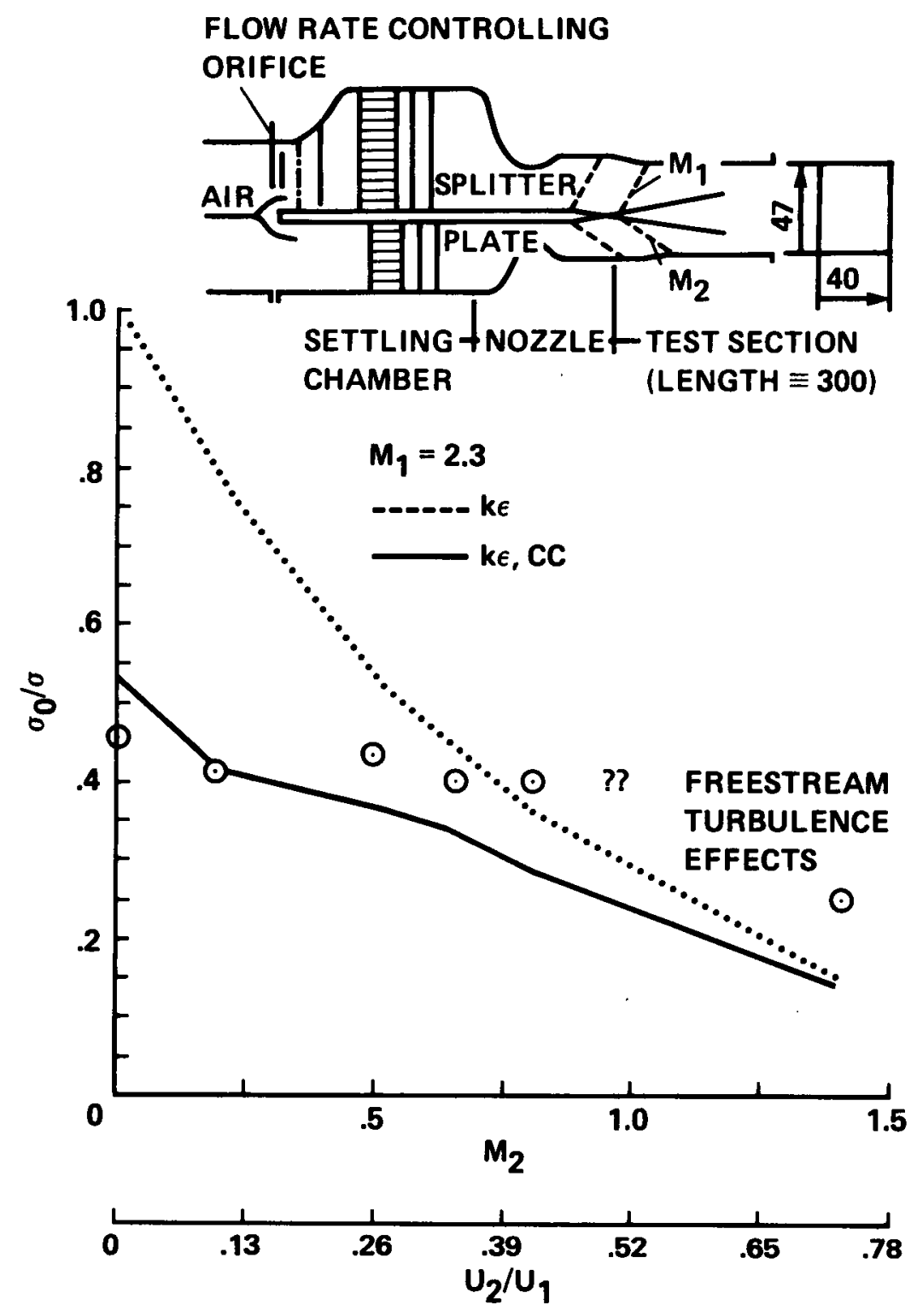

Fig. 23. Spreading rates for a two-stream compressible 2-D shear layer. 


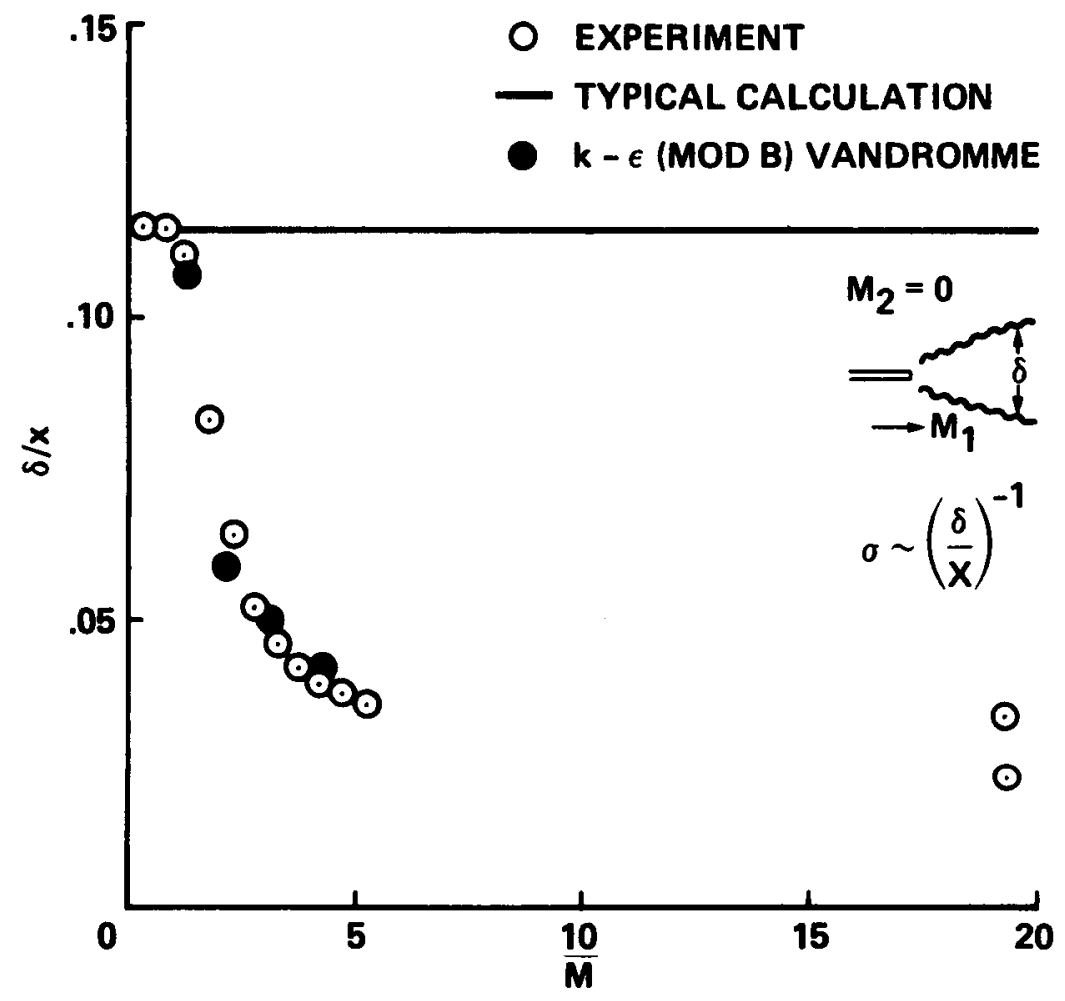

Fig. 24. Spreading rates for compressible 2-D shear layers. 


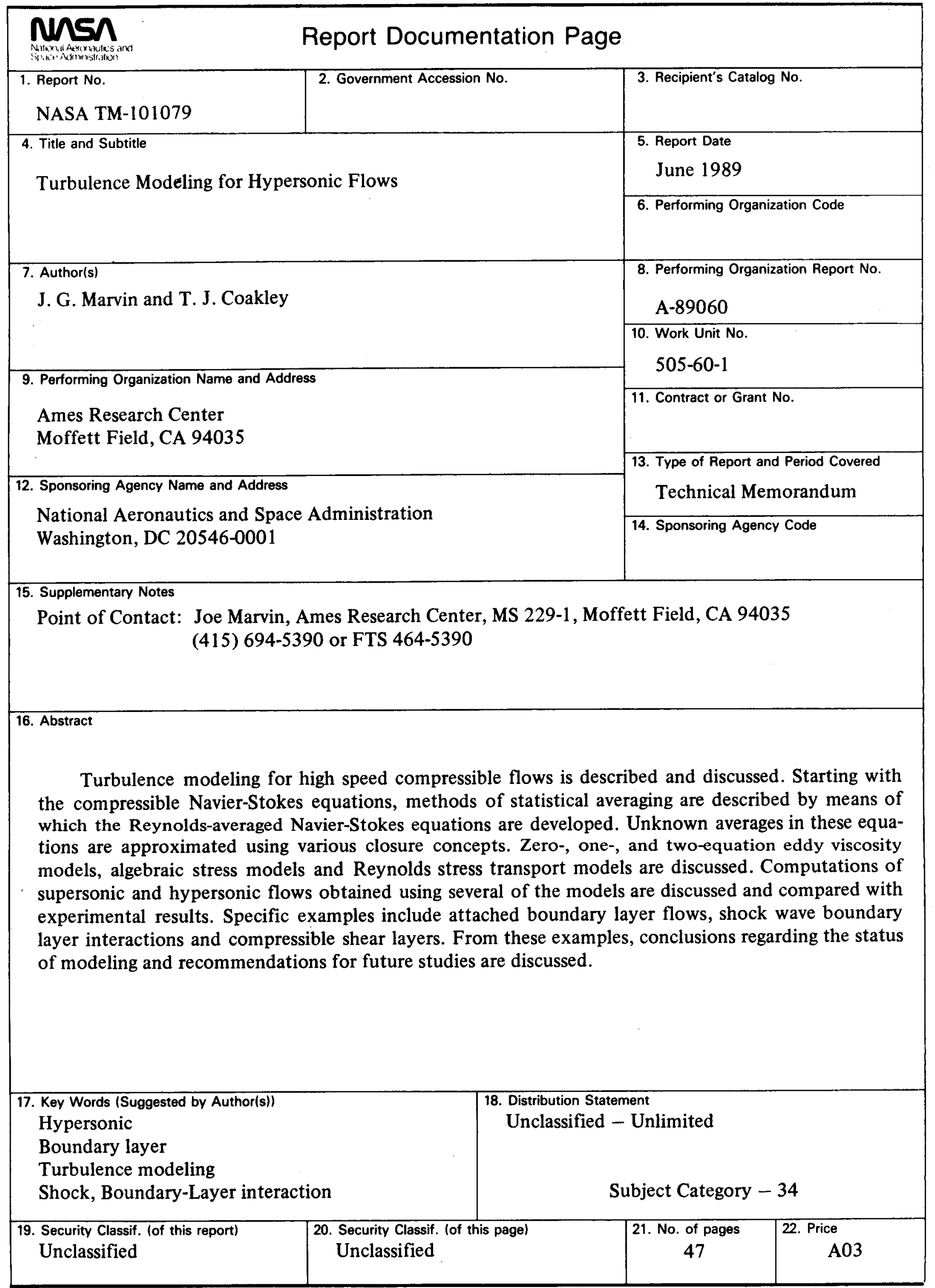

